\title{
Stability of Operator-Valued Truncated Moment Problems
}

\author{
L. Lemnete-Ninulescu \\ Department of Mathematics, Politechnica University of Bucharest, Bucharest, Romania \\ Email: luminita_lemnete@yahoo.com
}

Received January 8, 2013; revised February 10, 2013; accepted February 17, 2013

Copyright (C) 2013 L. Lemnete-Ninulescu. This is an open access article distributed under the Creative Commons Attribution License, which permits unrestricted use, distribution, and reproduction in any medium, provided the original work is properly cited.

\begin{abstract}
In this note a multidimensional Hausdorff truncated operator-valued moment problem, from the point of view of "stability concept" of the number of atoms of the obtained atomic, operator-valued representing measure for the terms of a finite, positively define kernel of operators, is studied. The notion of "stability of the dimension" in truncated, scalar moment problems was introduced in [1]. In this note, the concept of "stability" of the algebraic dimension of the obtained Hilbert space from the space of the polynomials of finite, total degree with respect to the null subspace of a unital square positive functional, in [1], is adapted to the concept of stability of the algebraic dimension of the Hilbert space obtained as the separated space of some space of vectorial functions with respect to the null subspace of a hermitian square positive functional attached to a positive definite kernel of operators. In connection with the stability of the dimension of such obtained Hilbert space, a Hausdorff truncated operator-valued moment problem and the stability of the number of atoms of the representing measure for the terms of the given operator kernel, in this note, is studied.
\end{abstract}

Keywords: Operator-Valued Positive-Definite Function; Unitary-Operator; Selfadjoint Operator; Joint Spectral Measure of a Commuting Tuple of Operators; Atomic Measure; Extension of Some Hermitian; Square; Positive Functional

\section{Introduction}

The study of scalar truncated moment problems is the subject of many remarkable papers such as: [1-3]. In [2], the problem of finding scalar atomic representing measure for the terms of a finite scalar sequence of complex numbers as multidimensional moment terms is studied. In [2], with the Hankel matrix $M_{\gamma}=\left\{\gamma_{\alpha+\beta}\right\}_{\alpha, \beta \in \mathbb{N}^{p}} \subset \mathrm{C}^{p}$ with rank $r$, the necessary and sufficient existence condition of an atomic representing measure with exactly $r$ atoms for the sequence $\left\{\gamma_{\alpha+\beta}\right\}_{\alpha, \beta \in \mathbb{N}^{p}}$ is the existence of a "flat extension". A "flat extension" is a rank preserving, nonnegative extension $M_{\gamma^{\prime}}$ of $M_{\gamma}$, associated with a larger moment sequence $\gamma^{\prime}$. A main result in [2], establishes some algebraic relations between the condition in which the support of the representing measure of the given sequence $\gamma$ is contained in the algebraic variety of zerous of a suitable polynomial and the dependence relations established between the columns of the Hankel matrix $M_{\gamma}$. These relations are expressed, also, as zerous of the mentioned polynomial. In [1], the concept of "flat extension" of the Hankel matrix of truncated sca- lar multidimensional moment sequence is substituted with the concept of "dimension stability" of the algebraic dimension of the Hilbert space obtained as the separation of the space of scalar polynomials with total degree $m$, with respect to the null subspace of a unital, square positive functional, the Riesz functional. The Riesz functional is in bijection with a moment functional, positive on the cone of sums of squares of real polynomials. The stability condition of the algebraic dimension of such Hilbert space affords in [1] an algebraic condition for obtaining some commutative tuple of selfadjoint operators, defined on the Hilbert space of stable dimension. In the same time, in [1], by extending the Riesz functional on the whole space of polynomials, using the functional calculus of the constructed commutative selfadjoint tuple, the arbitrary powers of it are organized as a $\mathrm{C}^{*}$ algebra of the same stable dimension. The problem of stability of the algebraic dimension of some Hilbert space obtained in this way is naturally connected, via the existence of a commuting tuple of self adjoint operators, with that of solving a scalar truncated multidimensional moment problem. The representing measure of the finite dimensional moment sequence is, in [1], the spectral atomic joint measure associated with the constructed commuta- 
tive selfadjoint tuple, and has the same number of atoms as the stable algebraic dimension. Truncated operatorvalued problems is the subject in papers [4-7], to quote only a few of them. In [7] a Hausdorff truncated unidimensional operator-valued moment problem is studied. For obtaining the representing measure the Kolmogorov's decomposition theorem is used. The given positive kernel of operators in [7] acts on an arbitrary, separable Hilbert space, all operators are linear independent, also the number of operators is arbitrary, even or odd.

In the present note, the stability dimension concept in [1], in the following way is adapted: a positive finite operator-valued kernel acting on a finite dimensional Hilbert space is given; a hermitian square positive functional on the space of vectorial functions, via the given kernel, is introduced. The restrictions of the hermitian square positive functional to some subspaces of the vectorial functions are considered. The separation spaces with respect to the null subspaces of these hermitian square positive restricted functionals are obtained. The stability dimensional condition for the obtained Hilbert spaces, in the same way as in [1], affords a construction of a commuting tuple of selfadjoint oparators, defined on the Hilbert space of the stable dimension. The obtained commuting tuple of selfadjoint operators, produced an integral representing joint spectral measure of all powers of the tuple. Via Kolmogorov's theorem of decomposition of positive operator kernels, a representing positive operator-valued measure as Hausdorff truncated multidimensional moment sequence for all terms in the given kernel is obtained. The first terms of the given kernel, in number equal with "d"- the stable dimension, are linear independent, are integral represented with respect to an atomic operator valued measure with exactely "d" atoms, the remainder terms in the kernel are integral represented with respect to the same measure and the same number of atoms as the first one. The possibility of extension of the given operator sequence with preserving the "stability condition", as well as the number of atoms of the representing measure is also analysed. In the present note, the number of operators in the given kernel is only even.

In this note, in Section 3 to a positive-definite kernel of operators a square positive functional is attached. The Hilbert spaces obtained as the quotient of some finite dimensional spaces and subspaces of vectorial functions with respect to the null spaces associated with the square positive functional and its restrictions are constructed. The problem of stability of the dimension of the Hilbert spaces in Section 3 and its implications in solving multidimensional, truncated Hausdorff operator-valued moment problems in Section 4, in this note is analysed.

\section{Preliminaries}

When $p \in \mathrm{N}^{*}, q \in \mathrm{N}^{p}, q=\left(q_{1}, \cdots, q_{p}\right)$ and $t=\left(t_{1}, \cdots, t_{p}\right) \in \mathrm{R}^{p}$, the $p$-dimensional real variable, are arbitrary, we denote with $t^{q}=t^{q_{1}}, \cdots, t^{q_{p}}$; the addition and substraction in $\mathrm{N}^{p}$ are considered on components. For $\mathrm{H}$ an arbitrary Hilbert space, $B(\mathrm{H})$ represents the algebra of linear, bounded operators on $\mathrm{H}$, for $A=\left(A_{1}, \cdots, A_{p}\right)$ a commuting tuple of multioperators, $A_{i} \in B(\mathrm{H})$ for all $1 \leq i \leq p$, we denote with $A^{q}=A^{q_{1}} \circ \cdots \circ A^{q_{p}}$. For an arbitraty $n \in \mathrm{N}^{p}$, the function $\delta_{n}: \mathrm{N}^{p} \rightarrow\{0,1\} \subset \mathrm{R}$, is:

$$
\delta_{n k}=\prod_{i=1}^{p}\left[\delta_{n_{i} k_{i}}\right]=\left\{\begin{array}{l}
1, k=n \\
0, k \neq n
\end{array}\right.
$$

with $\delta_{n, k_{i}}$ the Kronecker symbol. For $N \in \mathrm{N}^{*}$, we consider the spaces of vectorial functions:

$$
\begin{aligned}
F_{N}=\left\{f:\{0,1, \cdots, N\}^{p} \rightarrow \mathrm{H},\right. \\
\\
\left.\quad f(\cdot)=\sum_{n \in\{0, \cdots, N\}^{p}} \delta_{n} \cdot f(n), f(n) \in \mathrm{H}\right\}
\end{aligned}
$$

and, for each integer $k<N$, we denote with

$$
F_{k}=\left\{f:\{0,1, \cdots, k, \cdots, N\}^{p} \rightarrow \mathrm{H}\right\}
$$

with $f(q)=0$ for all multiindices $q \in \mathrm{N}^{p}$, with at least one indices $q_{j}>k$, the C-vector subspaces of $F_{N}$. For $e_{i}=(0, \cdots, 1, \cdots, 0) \in \mathrm{N}^{p}, 1 \leq i \leq p$ we also use the same function, $\delta_{e_{i}}$. defined by:

$$
\begin{aligned}
& \delta_{e_{i}}: Z^{p} \rightarrow\{0,1\}, \\
& \delta_{e_{i} k}=\left\{\begin{array}{l}
1, k=e_{i}=(0, \cdots, 1, \cdots 0) \text { on, } i \text { position } \\
0, k \neq e_{i} \in \mathrm{N}^{p}
\end{array}\right.
\end{aligned}
$$

and, for all

$$
\begin{array}{r}
f \in F_{N}^{i}=\left\{f:\{0, \cdots, N\}^{p} \rightarrow \mathrm{H}, f(\cdot)=\sum_{n} \delta_{n} \cdot f(n),\right. \\
n \in\{0, \cdots, N\} \times \cdots \times\{0, \cdots, N-1\} \times \cdots \times\{0, \cdots, N\}\},
\end{array}
$$

we define the convolutions $f * \delta_{e_{i}} \in F_{N}$ as

$$
\begin{aligned}
{\left[f * \delta_{e_{i}}\right](n)=} & \sum_{k \in \mathbb{Z}^{p}}\left(f(n-k) \cdot \delta_{e_{i} k}\right), \\
& n \in\{0, \cdots, N\}^{p}, \forall 1 \leq i \leq p,
\end{aligned}
$$

that is $f * \delta_{e_{i}}=\sum_{n \in\{0, \cdots, N\} \times \cdots \times\{0, \cdots, N-1\} \times \cdots \times\{0, \cdots, N\}} \delta\left({ }_{n+e_{i}}\right) f(n)$.

\section{Hilbert Spaces Associated with Finite Positive Operator Valued Kernels; Algebraic Prerequisite}

We consider for $N \in \mathrm{N}^{*}$ an operator kernel 


$$
\begin{array}{r}
\Gamma_{N}=\left\{\Gamma_{m} \in B(\mathrm{H}), m=\left(m_{1}, \cdots, m_{p}\right)\right. \\
\text { with } \left.0 \leq m_{i} \leq 2 N, 1 \leq i \leq p\right\},
\end{array}
$$

subject on the condition $\Gamma_{m}=\Gamma_{m}^{*}$ for all $m$ with $m_{i} \leq 2 N, 1 \leq i \leq p, \Gamma_{0}=\operatorname{Id}_{\mathrm{H}}$, acting on a finite dimensional complex vector space $H$, positively defined. That is the kernel $\Gamma_{N}$ satisfies the condition

(A) $\sum_{m, n \in\{0,1, \cdots, N\}^{p}}\left\langle\Gamma_{n+m} x_{m}, x_{n}\right\rangle_{\mathrm{H}} \geq 0$

for all sequences $\left\{x_{n}\right\}_{n \in\{0,1, \cdots, N\}}^{p}$ in H. When

$$
F_{N}=\left\{f:\{0,1, \cdots, N\}^{p} \rightarrow \mathrm{H}, f=\sum_{n \in\{0,1, \cdots, N\}^{p}} \delta_{n} \cdot f(n)\right\}
$$

is the $\mathrm{C}$-vector space of functions defined on $\{0,1, \cdots, N\}^{p}$ with vectorial values, we consider the kernel $\Gamma_{N}$ as a double indexed, simmetric one:

$$
\begin{aligned}
& \Gamma_{N}:\{0,1, \cdots, N\}^{p} \times\{0,1, \cdots, N\}^{p} \rightarrow B(\mathrm{H}), \\
& \Gamma_{n+m}=\Gamma(n, m) .
\end{aligned}
$$

With the aid of $\Gamma_{N}$, we introduce the hermitian, square positive functional

$$
\begin{aligned}
& \Lambda_{N}^{\Gamma}: F_{N} \times F_{N} \rightarrow \mathrm{C}, \\
& \Lambda_{N}^{\Gamma}(f, g)=\sum_{m, n \in\{0,1, \cdots, N\}^{p}}\left\langle\Gamma_{n+m} f(m), g(n)\right\rangle_{\mathrm{H}} \geq 0 ;
\end{aligned}
$$

the order in $\{0,1, \cdots, N\}^{p}$ is the lexicographical one. From property A of the the kernel $\Gamma_{N}$, as well as from the properties of the scalar product in $\mathrm{H}, \Lambda_{N}^{\Gamma}$ satisfies the conditions:

1) $\Lambda_{N}^{\Gamma}$ is C-linear in the first argument.

2) $\overline{\Lambda_{N}^{\Gamma}(f, g)}=\Lambda_{N}(g, f)$, for all $f, g \in F_{N}$.

3) $\Lambda_{N}^{\Gamma}(f, f) \geq 0$, for all $f \in F_{N}$.

\section{Remarks 3.1.}

a) $\Lambda_{N}^{\Gamma}$ is a hermitian, square, positive functional on $F_{N} \times F_{N}$ it results that $\Gamma_{N}$ satisfies the Cauchy-Buniakovski-Schwartz inequality, respectively:

$$
\left|\Lambda_{N}^{\Gamma}(f, g)\right| \leq \Lambda_{N}^{\Gamma}(f, f)^{\frac{1}{2}} \Lambda_{N}^{\Gamma}(g, g)^{\frac{1}{2}}, \forall f, g \in F_{N} .
$$

b) Because of the construction of the hermitian functional $\Gamma_{N}$ and the simmetry of the kernel $\Gamma_{N}, \Lambda_{N}^{\Gamma}$ satisfies the equalities:

$$
\begin{aligned}
& \Lambda_{N}^{\Gamma}\left(f * \delta_{e_{i}}, g\right)=\Lambda_{N}\left(f, g * \delta_{e_{i}}\right), \\
& \forall f, g \in F_{N-1}, \forall 1 \leq i \leq p .
\end{aligned}
$$

Definition 3.2. A functional $\Lambda_{N}^{\Gamma}$ defined on $F_{N} \times F_{N}$ with properties 1)-3) is called a hermitian, square positive functional on $F_{N}$.

Let $T_{N}$ be the subset in $F_{N}$, defined as
$T_{N}=\left\{f \in F_{N}\right.$ with $\left.\Lambda_{N}^{\Gamma}(f, f)=0\right\}$. If follows, using the Cauchy-Buniakowski-Schwarz inequality, that if $f_{1}, f_{2} \in$ $T_{N}$, we have also $\Lambda_{N}^{\Gamma}\left(\alpha_{1} f_{1}+\alpha_{2} f_{2}, \alpha_{1} f_{1}+\alpha_{2} f_{2}\right)=0$, that is $T_{N} \subset F_{N}$ is a vector subspace in $F_{N}$. The map

$F_{N} \ni f \rightarrow \Lambda_{N}^{\Gamma}(f, f)^{\frac{1}{2}} \in \mathrm{C}$ is a seminorm on $F_{N}$. Because $\mathrm{H}$ is a finite dimensional complex vector space, $F_{N}$ is the same. We consider the separated space of $F_{N}$ with respect to $T_{N}$, that is, in this case, the quotient space $K_{N}=F_{N} / T_{N}$. Obviously, in finite dimensional case for $F_{N}, K_{N}$ is a finite dimensional Hilbert space with the norm $\|\hat{p}\|_{K_{N}}=\Lambda_{N}^{\Gamma}\left(p+T_{N}, p+T_{N}\right)^{\frac{1}{2}}$ for an arbitrary $p \in F_{N}$. In the special case of the space $F_{N}=\left\{f:\{0,1, \cdots, N\}^{p} \rightarrow \mathrm{H}\right\}$, we have $K_{N}=F_{N} / T_{N}=V_{n \in\{0, \cdots, N\}^{p}, v_{j} \in \mathrm{H}}\left[h_{n} v_{j}\right]$ where $\left[h_{n} v_{j}\right]$ represents the class in $K_{N}$ of the function $F_{N} \ni \delta_{n} \cdot v_{j}:\{0,1, \cdots, N\}^{p} \rightarrow \mathrm{H}, \delta_{n} \cdot v_{j}=\prod_{i=1}^{p}\left[\delta_{n_{i} k_{i}}\right] v_{j}, \delta_{n_{i} k_{i}}$ the usual Kroneker symbol, $\left\langle v_{1}, \cdots, v_{k}\right\rangle=\mathrm{H}$ the basis of $\mathrm{H}$ and $\mathrm{V}$ represents the linear span of these elements. When $0 \leq k<N$, we consider the vector subspaces $F_{k} \subset F_{N}$, with

$$
\begin{gathered}
F_{k}=\left\{f:\{0, \cdots, N\}^{p} \rightarrow \mathrm{H}, f(q)=0\right. \text { for all multiindices } \\
q=\left(q_{1}, \cdots, q_{p}\right) \in\{0,1, \cdots, N\}^{p} \text { with at least } \\
\text { one indices } \left.q_{i}>k, 1 \leq i \leq p\right\}
\end{gathered}
$$

and the restriction $\left.\Lambda_{N}^{\Gamma}\right|_{F_{k} \times F_{k}}$, restriction denoted with $\Lambda_{k}^{\Gamma}$. The functional $\Lambda_{k}^{\Gamma}$ is also a hspf on $F_{k}$, consequently it has properties 1), 2), 3) and a), b) from $\mathrm{Re}$ marks 3.1. Setting

$$
\begin{aligned}
T_{k} & =\left\{f \in F_{k}, \Lambda_{k}^{\Gamma}(f, f)=0\right\} \\
& =F_{k} \cap T_{N} \subset T_{N},
\end{aligned}
$$

it results that $T_{k}$ is a vector subspace in $F_{k}$ and in $T_{N}$. We denote with $K_{k}$ the Hilbert space $K_{k}=F_{k} / T_{k}$ with the norm

$$
\|\hat{f}\|_{K_{k}}=\left\langle f+T_{k}, f+T_{k}\right\rangle^{\frac{1}{2}}=\Lambda_{k}(f, f)^{\frac{1}{2}},
$$

for all $f \in F_{k}$. Because $T_{k} \subset T_{N}, F_{k} \subset F_{N}$, it results that also $K_{k} \subset K_{N}$ and there is the natural inclusion map $J_{k, N}: K_{k} \rightarrow K_{N},\left.\hat{f}\right|_{K_{k}}=f+\left.T_{k} \rightarrow \hat{f}\right|_{K_{N}}=f+T_{N}$. The inclusion map $J_{k, N}$ is injective one. Indeed, when $J_{k, N}\left(\hat{f}_{1}\right)=J_{k, N}\left(\hat{f}_{2}\right), \quad f_{1}, f_{2} \in F_{k}$, it results $f_{1}+T_{N}=$ $f_{2}+T_{N}$, that is $f_{1}-f_{2} \in T_{N}$. The elements $f_{1}, f_{2}$ are in $F_{k}$, consequently 


$$
\Lambda_{k}^{\Gamma}\left(f_{1}-f_{2}, f_{1}-f_{2}\right)=\Lambda_{N}^{\Gamma}\left(f_{1}-f_{2}, f_{1}-f_{2}\right)=0,
$$

that is $f_{1}-f_{2} \in T_{K}$ or $\left.\hat{f}_{1}\right|_{F_{k}}=\left.\hat{f}_{2}\right|_{F_{k}}$ and $\left\|\hat{f}_{1}\right\|_{F_{k}}=\left\|\hat{f}_{2}\right\|_{F_{N}}$, $i=1,2$. We are interested in conditions in which $J_{k, N}$ is an isomorphism of vectorial spaces and $J_{k, N}\left(K_{k}\right)=$ $K_{N}$. Because all Hilbert spaces $K_{l}, 0 \leq l \leq N$, obtained in this way are finite dimensional, in case $J_{k, N}$ is an isomorphism of vectorial spaces, $K_{k}$ and $K_{N}$ have the same algebraic dimension. In connection with the problem of the stability of the algebraic dimension for the Hilbert spaces obtained as quatient of some vectorialvalued spaces of functions, we are interested in finding operator-valued atomic representing measure for the terms of $\Gamma_{N}$. For such operator-valued, representing atomic measure, the stable number of atoms for the representations of the operators $\tilde{\Gamma}=\left\{\Gamma_{m}\right\}_{m \in\{0,1, \cdots, N-1\}^{p}} \subset \Gamma_{N}$, is the same with that in $\Gamma_{N}^{\prime} \mathrm{S}$ integral representations. Such studies are the subject of truncated Hausdorff operator-valued moment problems. The concept of stability of the algebraic dimension of the Hilbert space obtained by separating the space of scalar polynomials with finite total degree with respect to an unital square positive functional (the Riesz functional), was introduced in [1]. The concept of stability of the algebraic dimension appears in [1] in the frame of extending some commuting tuple of selfadjoint operators which were intended to get the joint spectral representing measure for the terms of a Hausdorff truncated scalar moment sequences. The concept of stability of the algebraic dimension in [1] is an alternate, geometric aspect of that of "flatness" in Fialkow's and Curto's paper [2,3] regarding the truncated scalar moment problems.

We adapt and reformulate the concept of stability of the dimension concerning unital square positive functionals on space of scalar polinomyals in [1], to hermitian, square positive functionals associated with positive operator-valued kernels in order to solve operator-valued, truncated, Hausdorff moment problems via Kolmogorov's theorem of decomposition of such kernels. The problem to obtain operator-valued positive operator representing measure for truncated, trigonometric and Hausdorff operator-valued moment problems via Kolmogorov's theorem of decomposition of positive operator kernels were solved in [7].

The classical Kolmogorov's theorem of the decomposition of positive kernels states:

"Let $\Gamma: S \times S \rightarrow L(\mathrm{H})$ a nonnegative-definite function where $S$ is a set and $\mathrm{H}$ a Hilbert space, namely $\sum_{i, j=1}^{n}\left\langle\Gamma\left(s_{i}, s_{j}\right) x_{j}, x_{i}\right\rangle \geq 0$ for any finite number of points $s_{1}, \cdots, s_{n} \in S$ and any vectors $x_{1}, \cdots, x_{n} \in \mathrm{H}$. In this case there exists a Hilbert space $K$ (essentialy unique) and $a$ function $h: S \rightarrow B(\mathrm{H}, K)$ such that $\Gamma(l, m)=h(m)^{*} h(l)$ for any $l, m \in S$."

\section{Dimension Stability and Consequences in Truncated, Hausdorff, Multidimensional, Operator-Valued Moment Problems}

Let $N, p \in \mathrm{N}^{*}$,

$$
\begin{gathered}
\Gamma_{N}=\left\{\Gamma_{m} \in B(\mathrm{H}), m=\left(m_{1}, \cdots, m_{p}\right) \in \mathrm{N}^{p},\right. \\
\left.m_{i} \leq 2 N, 1 \leq i \leq p\right\}
\end{gathered}
$$

be an operator kernel with $\Gamma_{m}=\Gamma_{m}^{*}$ and $\Gamma_{0}=\mathrm{Id}_{\mathrm{H}}$, positively defined, acting on the finite dimensional Hilbert space $\mathrm{H}$, that is $\Gamma_{N}$ satisfies condition (A) from Section 3; we consider the vector space

$$
F_{N}=\left\{f:\{0, \cdots, N\}^{p} \rightarrow \mathrm{H}, f(\cdot)=\sum_{N \in\{0, \cdots, N\}^{p}} \delta_{n} \cdot f(n)\right\}
$$

and $\Lambda_{N}: F_{N} \times F_{N} \rightarrow \mathrm{C}$,

$$
\Lambda_{N}^{\Gamma}(f, g)=\sum_{n, m \in\{0, \cdots, N\}^{p}}\langle\Gamma(n, m) f(m), g(n)\rangle_{\mathrm{H}},
$$

the associated hspf with $\Gamma_{N}$. We consider the set $T_{N}=\left\{f \in F_{N}, \Lambda_{N}(f, f)=0\right\}$. Because $\Lambda_{N}^{\Gamma}$ satisfies Cauchy-Buniakovski-Schwarz inequality, $T_{N}$ is a vector subspace in $F_{N}$, and also

$$
T_{N}=\left\{g \in F_{N} \text { with } \Lambda_{N}^{\Gamma}(g, f)=0, \forall f \in F_{N}\right\}
$$

and $K_{N}=F_{N} / T_{N}=\left\{f+T_{N}, f \in F_{N}\right\}$ is a finite dimensional Hilbert space, with the norm

$\|\hat{f}\|_{K_{N}}=\Lambda_{N}^{\Gamma}\left(f+T_{N}, f+T_{N}\right)^{\frac{1}{2}}$. The space $K_{N}$ is refered as the Hilbert space obtained via the hspf $\Lambda_{N}^{\Gamma}$.

For every $l$ with $0 \leq l<N$, and

$$
\begin{gathered}
F_{l}=\left\{f:\{0, \cdots, N\}^{p} \rightarrow \text { H with } f(q)=0\right. \text { for all } \\
\\
q=\left(q_{1}, \cdots, q_{p}\right) \text { with at least one indices } \\
\left.q_{i}, l<q_{i} \leq N\right\},
\end{gathered}
$$

we consider the restriction $\Lambda_{l}=\left.\Lambda_{N}\right|_{F_{1} \times F_{l}}$ The functional $\Lambda_{l}$ is also a hspf on $F_{l}$ and satisfies conditions 1), 2), 3) and a), b) in Remark 3.1. The subset

$$
T_{l}=\left\{f \in F_{l}, \Lambda_{l}=\left\{f \in F_{l}, \Lambda_{l}(f, f)=0\right\}\right\}=T_{N} \cap F_{l}
$$

is obviously a subspace in $T_{N}$ and also in $F_{l}$. Consequently, the Hilbert space $K_{l}=F_{l} / T_{l}$ with respect to the norm $\|\hat{f}\|_{K_{l}}=\Lambda_{l}(f, f)^{\frac{1}{2}}$ is defined via the hspf $\Lambda_{l}$ and because $T_{l} \subseteq T_{N} \cap F_{l}$ is a vector subspace in $T_{N}$, the natural inclusion map $J_{l, N}: K_{l} \rightarrow K_{N}, \widehat{f \mid K_{l}} \rightarrow \widehat{f \mid K_{N}}$, 
$f+T_{l} \rightarrow f+T_{N}$ for $f \in F_{l}$ is an isometry. For $l=N$, we have $J_{N, N}: K_{N} \rightarrow K_{N}, J_{N, N}=\operatorname{Id}_{K_{N}}$. In the same way, for all $k \leq l \leq N$ we have $T_{k} \subset T_{l}, F_{k} \subset F_{l}$ and consequently there exists the naturaly isometries $J_{k, l}: K_{k} \rightarrow K_{l}$.

If we have an operator-valued kernel $\Gamma=\left\{\Gamma_{m}\right\}_{m \in \mathbb{N}^{p}}$ acting on a finite dimensional Hilbert space $\mathrm{H}$, subject on $\Gamma_{m}=\Gamma_{m}^{*}$ for all $m \in \mathrm{N}^{p}, \Gamma_{0}=\operatorname{Id}_{\mathrm{H}}$, such that

$\sum_{m, n \in \mathrm{I} \text { finite }}\left\langle\Gamma_{n+m} x_{m}, x_{n}\right\rangle_{\mathrm{H}} \geq 0$ for all sequences $\left\{x_{n}\right\}_{n} \subset \mathrm{H}$ with finite support, considering the vector space

$$
F_{\infty}=\left\{f: \mathrm{N}^{p} \rightarrow \mathrm{H}, f \text { with finite support }\right\},
$$

$\Gamma_{n+m}=\Gamma(n, m)$ the associated functional

$\Lambda_{\infty}^{\Gamma}: F_{\infty} \times F_{\infty} \rightarrow \mathrm{C}, \quad \Lambda_{\infty}^{\Gamma}(f, g)=\sum_{m, n \in \mathrm{I}}\left\langle\Gamma_{n, m} f(m), g(n)\right\rangle_{\mathrm{H}}$,

with $\mathrm{I} \subset \mathrm{N}^{p}$ finite, the map $\Lambda_{\infty}^{\Gamma}$ is naturally a hspf on $F_{\infty} \times F_{\infty}$, with properties 1), 2), 3) and a), b) from Remark 3.1. Similar constructions of the Hilbert spaces $\left\{K_{l}\right\}_{l=0}^{+\infty}$ as well as for the isometries $\left\{J_{k, l}\right\}_{0<k \leq l<+\infty}$ can be done.

Definition 4.1. Let $\Gamma_{N}=\left\{\Gamma_{m}\right\}_{m \in \mathbb{N}^{p}} \in B(\mathrm{H}), n_{i} \leq 2 N$, $1 \leq i \leq p, \quad \Gamma_{n}=\Gamma_{n}^{*}, \quad \Gamma_{0}=\mathrm{Id}_{\mathrm{H}}$ be a positive definite kernel, $\Lambda_{N}^{\Gamma}: F_{N} \times F_{N} \rightarrow \mathrm{C}$ the hspf associated with $\Gamma_{N}$ and $\left\{K_{l}\right\}_{0<1<N}^{\Gamma}$ the Hilbert spaces built via $\Lambda_{N}^{\Gamma}$ and $J_{l-1, l}: K_{l-1} \rightarrow K_{l}, 0 \leq l \leq N-1$ the associated isometries. If for some $k \in\{0,1, \cdots, N-1\}$ the injective map $J_{k-1, k}$ is also surjective, that is $J_{k-1, k}\left(K_{k-1}\right)=K_{k}$ we say that $\Lambda_{N}^{\Gamma}$ and the kernel $\Gamma_{N}$ is dimensionally stable (stable) at $k$.

The kernel $\left\{\Gamma_{n}^{\infty}\right\}_{n \in \mathrm{N}^{p}}$ is called dimensionally stable if there exists integers $0<l<N<+\infty$ such that the kernel $\tilde{\Gamma}_{N}=\left\{\Gamma_{n} \in \Gamma^{\infty}\right.$, with $\left.n_{i} \leq 2 N, 1 \leq i \leq p\right\}$ is stable at $0<l \leq N-1$, respectively $\Lambda_{N}^{\Gamma}=\left.\Lambda_{\infty}^{\Gamma}\right|_{F_{N} \times F_{N}}$ is stable at $l$.

Remark 4.2. c) Let $\Gamma_{N}=\left\{\Gamma_{m}\right\}_{m \in N^{p}} \in B(\mathrm{H}), n_{i} \leq 2 N$, be a positive definite kernel, $\Lambda_{N}^{\Gamma}: F_{N} \times F_{N} \rightarrow \mathrm{C}$ the hspf associated with $\Gamma_{N}$ stable at $(N-1)$ and $\left\{K_{l}\right\}_{0 \leq 1 \leq N}$ the Hilbert spaces built via $\Lambda_{N}^{\Gamma} ;\left(J_{N-1, N}\right.$ are bijective maps, $J_{N-1, N}\left(K_{N-1}\right)=K_{N}$. ) In this case, the maps $M_{j}: K_{N-1} \rightarrow K_{N}$,

$$
M_{j}\left(f+T_{N-1}\right)=\sum_{n \in\{0,1, \cdots, N-1\}^{p}} \delta_{n+e_{j}} \cdot f(n)+T_{N},
$$

when $\hat{f}=\sum_{n \in\{0,1, \cdots, N-1\}^{p}} \delta_{n} \cdot f(n)+T_{N-1}$, and $e_{j}=\left(\delta_{k j}\right)_{k=0}^{p}$,

for all $1 \leq j \leq p$ are correctely defined.

Indeed, let $g=\sum_{n \in\{0,1, \cdots, N-1\}^{p}} \delta_{n} \cdot g(n)$ be such that

$(g-f) \in T_{N-1}$; we shall prove that $M_{j} f-M_{j} g \in T_{N}$. If $M_{j} f=\sum_{n \in\{0,1, \cdots, N-1\}^{p}} \delta_{n+e_{j}} \cdot f(n) \in F_{N}$ and $\Gamma_{N}$ is stable at $(N-1), J_{N-1, N}$ is an isomorphism of vectorial spaces, exists then $h \in F_{N-1}$ such that $M_{j}(f-g)-h \in T_{N}$. In this case, using property a) and b) in Remarks 3.1 for the kernel $\Gamma$ we have:

$$
\begin{aligned}
& \left|\Lambda_{N}^{\Gamma}\left(M_{j}(f-g), h\right)\right|=\left|\Lambda_{N}^{\Gamma}\left((f-g), M_{j} h\right)\right| \\
& \leq \Lambda_{N}^{\Gamma}((f-g), f-g)^{\frac{1}{2}} \Lambda_{N}^{\Gamma}\left(M_{j} h, M_{j} h\right)^{\frac{1}{2}}=0 .
\end{aligned}
$$

Also, using the Cauchy-Schwartz inequality,

$$
\begin{aligned}
& \left|\Lambda_{N}^{\Gamma}\left(M_{j}(f-g),\left(M_{j}(f-g)-h\right)\right)\right| \\
\leq & \Lambda_{N}^{\Gamma}\left(\left(M_{j}(f-g)\right)-h\right), \\
& \quad\left(\left(M_{j}(f-g)\right)-h\right)^{\frac{1}{2}} \Lambda_{N}^{\Gamma}(l, l)^{\frac{1}{2}}=0 ;
\end{aligned}
$$

where we have denoted with $l=\left(M_{j}(f-g)\right)$.

From (4), (5), we have:

$$
\begin{aligned}
& \Lambda_{N}^{\Gamma}\left(M_{j}(f-g), M_{j}(f-g)\right) \\
= & \Lambda_{N}^{\Gamma}\left(M_{j}(f-g), M_{j}(f-g)-h\right) \\
& +\Lambda_{N}^{\Gamma}\left(M_{j}(f-g), h\right)=0 .
\end{aligned}
$$

It results, that $M_{j} f-M_{j} g \in T_{N}$. The maps $M_{j}: K_{N-1} \rightarrow K_{N}, g+T_{N-1} \rightarrow M_{j}\left(g+T_{N-1}\right)$,

$$
\sum_{n \in\{0, \cdots, N-1\}} \delta_{n} \cdot g(n)+T_{N-1} \rightarrow \sum_{n \in\{0, \cdots, N-1\}} \delta_{n+e_{j}} \cdot g(n)+T_{N}
$$

are correctely defined.

d) If we consider the subspaces

$$
\begin{aligned}
F_{N-1, N}^{1}=\{ & f:\{0, \cdots, N\}^{p} \rightarrow \mathrm{H}, f(\cdot)=\sum \delta_{n} \cdot f(n), \\
& n=\left(n_{1}, \cdots, n_{p}\right), n_{1} \in\{0,1, \cdots, N\}, \\
& \left.n_{k} \in\{0, \cdots, N-1\}, k \neq n_{1}, f(n) \in \mathrm{H}\right\},
\end{aligned}
$$

$F_{N-1} \subset F_{N-1, N}^{1} \subset F_{N}$, also $\left.\Lambda_{N}^{\Gamma}\right|_{F_{N-1, N}^{1} \times F_{N-1, N}^{1}}$ the null spaces

$$
T_{N-1, N}^{1}=\left\{f \in F_{N-1 ; N}^{1}, \Lambda_{N}^{\Gamma}(f, f)=0\right\} \subset F_{N-1 ; N}^{1}
$$

of it. We have

$$
F_{N-1} \subset F_{N-1, N}^{1} \subset F_{N}, T_{N-1} \subset T_{N-1, N}^{1} \subset T_{N},
$$

it results,

$$
K_{N-1}=K_{N-1, N}^{0} \subset K_{N-1, N}^{1} \subset K_{N}=K_{N-1, N}^{p} .
$$

The same

$$
\begin{gathered}
F_{N-1, N}^{12}=\left\{f:\{0, \cdots, N\}^{p} \rightarrow \mathrm{H}, f(\cdot)=\sum \delta_{n} \cdot f(n),\right. \\
n=\left(n_{1}, \cdots, n_{p}\right), n_{1} \in\{0,1, \cdots, N\}, \\
\left.n_{k} \in\{0, \cdots, N-1\}, k \neq n_{1}, n_{2}, f(n) \in \mathrm{H}\right\}, \\
F_{N-1} \subset F_{N-1, N}^{1} \subset F_{N-1, N}^{12}, \text { and the null subspace } \\
T_{N-1, N}^{12}=\left\{f \in F_{N-1 ; N}^{12}, \Lambda_{N}^{\Gamma}(f, f)=0\right\} \subset F_{N-1 ; N}^{12}
\end{gathered}
$$


of it. We have also $T_{N-1 ; N}^{1} \subset T_{N-1 ; N}^{12}$ for the Hilbert quotient spaces we obtain

$$
\begin{aligned}
K_{N, N-1}^{0} & \subset K_{N-1 ; N}^{1}=F_{N-1 ; N}^{1} / T_{N-1 ; N}^{1} \\
& \subset F_{N-1 ; N}^{12} / T_{N-1 ; N}^{12}=K_{N-1 ; N}^{12} .
\end{aligned}
$$

In the same way, by recurrence, we obtain the vectorial subspaces

$$
\begin{aligned}
F_{N-1, N}^{12 \cdots k}=\{ & f:\{0, \cdots, N\}^{p} \rightarrow \mathrm{H}, f(\cdot)=\sum \delta_{n} \cdot f(n), \\
& n=\left(n_{1}, \cdots, n_{p}\right), n_{i} \in\{0,1, \cdots, N\}, \\
& i \in\{1,2, \cdots, k\}, n_{j} \in\{0, \cdots, N-1\}, \\
& j \in\{k+1, \cdots, p\}, f(n) \in \mathrm{H}\},
\end{aligned}
$$

the null subspaces

$$
T_{N-1, N}^{12 \cdots k}=\left\{f \in F_{N-1 ; N}^{12 \cdots k}, \Lambda_{N}^{\Gamma}(f, f)=0\right\} \subset F_{N-1 ; N}^{12 \cdots k}
$$

of it and the required quotient Hilbert space

$K_{N-1 ; N}^{12 \cdots k}=F_{N-1 ; N}^{12 \cdots k} / T_{N-1 ; N}^{12 \cdots k}$ for all $1 \leq k \leq p$. From the above construction, the inclusions of the Hilbert spaces

$$
\begin{aligned}
K_{N-1} & =K_{N-1, N}^{0} \subset K_{N-1 ; N}^{1} \subset K_{N-1}^{12} \\
& \subset K_{N-1 ; N}^{123} \subset \cdots \subset K_{N-1 ; N}^{12 \cdots p}=K_{N}
\end{aligned}
$$

and the naturally isometries

$$
\begin{gathered}
J_{N-1 ; N}^{k, k+1}: K_{N-1 ; N}^{1 \cdots k} \rightarrow K_{N-1 ; N}^{1 \cdots k+1}, \\
\hat{f}=f+T_{N-1 ; N}^{12 \cdots k} \rightarrow f+T_{N-1 ; N}^{12 \cdots k+1}
\end{gathered}
$$

are obtained, for all $0 \leq k \leq p-1$. Because $\Gamma_{N}$, respectively $\Lambda_{N}^{\Gamma}$ are stable at $N-1$, it results

$$
\begin{aligned}
& \operatorname{dim}_{\mathrm{C}} K_{N-1, N}^{0}=\operatorname{dim}_{\mathrm{C}} K_{N-1 ; N}^{12 \cdots k}=\operatorname{dim}_{\mathrm{C}} K_{N}=d, \\
& 0 \leq k \leq p-1 .
\end{aligned}
$$

Consequently all the isometries $J_{N-1 ; N}^{k, k+1}: K_{N-1 ; N}^{1 \cdots k} \rightarrow K_{N-1 ; N}^{1 \cdots k+1}$ are surjective one. Let as consider the operators:

$$
\begin{gathered}
M_{1, i}: K_{N-1 ; N}^{0} \rightarrow K_{N-1 ; N}^{1}, \\
\hat{f}=\sum \delta_{n} \cdot f(n)+T_{N-1}, \\
M_{1, i} \hat{f}=\sum \delta_{n+e_{i}} \cdot f(n)+T_{N}^{1}
\end{gathered}
$$

and

by

$$
A_{1, i}: K_{N-1 ; N}^{1} \rightarrow K_{N-1 ; N}^{1}
$$

$$
A_{1, i}=M_{1 i} \circ\left[J_{N-1^{\prime} N}^{0,1}\right]^{-1}, 1 \leq i \leq p
$$

$$
\begin{gathered}
A_{k, l} \circ A_{k, m}\left(\sum_{n \in(0,1, \cdots, N)^{k} \times\{0, \cdots, N-1\}^{p-k}} \delta_{n} \cdot f(n)+T_{N-1, N}^{12 \cdots k}\right) \\
\forall 0 \leq k \leq p-2 .
\end{gathered}
$$

$$
\begin{gathered}
M_{2 i}: K_{N-1 ; N}^{1} \rightarrow K_{N-1 ; N}^{12}, \\
M_{2, i} \hat{f}=\sum \delta_{n+e_{2}} \cdot f(n)+T_{N-1 ; N}^{12}
\end{gathered}
$$

and

$$
A_{2, i}^{2}: K_{N-1 ; N}^{12} \rightarrow K_{N-1 ; N}^{12}
$$

by $A_{2 i}=M_{2 i} \circ\left[J_{N-1 ; N}^{12}\right]^{-1}$. By recurrence the operators

$$
\begin{gathered}
M_{p, i}: K_{N-1 ; N}^{12 \cdots p-1} \rightarrow K_{N-1 ; N}^{12 \cdots p}, 1 \leq i \leq p \\
f=\sum \delta_{n} \cdot f(n)+T_{N-1 ; N}^{12 \cdots p-1} \in K_{N-1 ; N}^{12 \cdots p-1} ; \\
M_{p i}\left(f+T_{N-1 ; N}^{12 \cdots p-1}\right)=\sum \delta_{\left(n+e_{i}\right)} \cdot f(n)+T_{N}
\end{gathered}
$$

and

$$
\begin{gathered}
A_{p i}: K_{N-1 ; N}^{12 \cdots p} \rightarrow K_{N-1 ; N}^{12 \cdots p} ; \\
A_{p, i}=M_{p, i} \circ\left[J_{N-1, N}^{p-1, p}\right](-1), 1 \leq i \leq p .
\end{gathered}
$$

From the construction, immediately, it follows that

$$
J_{N-1 ; N}^{k, k+1} \circ M_{k-1, j}=M_{k, j} \circ J_{N-1 ; N}^{k-1 ; k} .
$$

The operators $M_{s, j}, 1 \leq j \leq p$ are correctely defined. Indeed, let $f=\sum_{n} \delta_{n} f(n) \in T_{N-1, N}^{1 \cdots s} \subset T_{N}$; we show that also $M_{s, j} f \in T_{N}^{1 \cdots s}$; from the stability condition, it exists $r \in F_{N-1}$ such that $M_{s, j} f-r \in T_{N}$ and, from CauchyBuniakovski-Schwartz and property b) of the kernel $\Gamma_{N}$, we have:

$$
\begin{aligned}
& \Lambda_{N}^{\Gamma}\left(M_{j} f, M_{j} f\right) \\
& =\Lambda_{N}^{\Gamma}\left(M_{j} f, M_{j} f-r\right)+\Lambda_{N}^{\Gamma}\left(M_{j} f, r\right) \\
& =\Lambda_{N}^{\Gamma}\left(M_{j} f, M_{j} f-r\right)+\Lambda_{N}^{\Gamma}\left(f, M_{j} r\right)=0 .
\end{aligned}
$$

That is the operators $M_{s, j}: K_{N-1, N}^{1 \cdots s-1} \rightarrow K_{N-1, N}^{1 \cdots s}$ are correctly defined. and extend the operators $M_{j}: K_{N-1} \leftrightarrow K_{N}$ to $K_{N-1, N}^{1 \cdots s}$ We apply (6) for computing

$$
\begin{aligned}
A_{k, l} \circ A_{k, m} & =M_{k-1, l} \circ\left[J_{N-1 ; N}^{k-1, k}\right]^{-1} \circ M_{k-1, m} \circ\left[J_{N-1 ; N}^{k-1, k}\right]^{-1} \\
& =\left[J_{N-1 ; N}^{k, k+1}\right]^{-1} \circ M_{k, l} \circ\left[J_{N-1 ; N}^{k, k+1}\right]^{-1} \circ M_{k, m} \\
& =\left[J_{N-1 ; N}^{k, k+1}\right]^{-1} \circ\left[J_{N-1 ; N}^{k+1, k+2}\right]^{-1} \circ M_{k+1, l} \circ M_{k, m} \\
& =\left[J_{N-1 ; N}^{k+2, k}\right]^{-1} \circ M_{k+1, l} \circ M_{k, m} .
\end{aligned}
$$

Consequently, it results 
where we have denoted

$$
\left[J_{N-1 ; N}^{k, k+2}\right]^{-1}=\left[J_{N-1 ; N}^{k, k+1}\right]^{-1} \circ\left[J_{N-1 ; N}^{k+1, k+2}\right]^{-1} .
$$

e) We consider the maps $A_{j}: K_{N} \rightarrow K_{N}$ defined by $A_{j}=M_{j} J_{N-1, N}^{-1}$, for $1 \leq j \leq p$. With $J_{N-1, N}$ the given isomorphism of vectorial spaces in case of $\Gamma_{N}$, stable at $N-1$ and with $M_{j}$, the linear operators $1 \leq i \leq p$ in c), the obtained $A_{j}$ operators are linear, correctely defined too.

Proposition 4.3. The linear operators $A_{j}: K_{N} \rightarrow K_{N}$, $A_{j}=M_{j} J_{N-1, N}^{-1}, 1 \leq j \leq p$ are selfadjoin on $K_{N}$ and the tuple $A=\left(A_{1}, \cdots, A_{p}\right)$ is a commuting multioperator on $K_{N}$.

Proof. From Remark 4.1. e) The operators $A_{j}: K_{N} \rightarrow K_{N}, A_{j}=M_{j} J_{N-1, N}^{-1}$, are linear, correctely defined on the Hilbert space $K_{N}$. We show that $A_{j}$ are selfadjoint one and commute; that is we verify $\left\langle A_{j}^{*} \hat{x}, \hat{y}\right\rangle_{K_{N}}=\left\langle A_{j} \hat{x}, \hat{y}\right\rangle_{K_{N}}$ and $A_{j} \circ A_{k}=A_{k} \circ A_{j}$ for all $\hat{x}, \hat{y} \in K_{N}, 1 \leq j, k \leq p$. Let $\hat{x}, \hat{y} \in K_{N}$ arbitrary,

$$
\begin{aligned}
& \hat{x}=\sum_{n \in\{0,1, \cdots, N\}^{p}} \delta_{n} \cdot x_{n}+T_{N}, \\
& \hat{y}=\sum_{n \in\{0,1, \cdots, N\}^{p}} \delta_{n} \cdot y_{n}+T_{N} .
\end{aligned}
$$

From the stability of $\Gamma_{N}$ and $\Lambda_{N}^{\Gamma}$ at $N-1, J_{N-1, N}$ is an isomorphism of vectorial spaces; it results that there exist

$$
\begin{gathered}
x^{\prime}=\sum_{n \in\{0,1, \cdots, N-1\}^{p}} \delta_{n} \cdot x_{n}, \\
y^{\prime}=\sum_{n \in\{0,1, \cdots, N-1\}^{p}} \delta_{n} \cdot y_{n}, x^{\prime}, y^{\prime} \in F_{N-1}
\end{gathered}
$$

with $x-x^{\prime}, y-y^{\prime} \in T_{N}$.

We have

$$
\begin{aligned}
& \left\langle A_{j}^{*} \hat{x}, \hat{y}\right\rangle_{K_{N}}=\left\langle A_{j}^{*}\left(\sum_{n \in\{0,1, \cdots, N\}^{p}} \delta_{n} \cdot x_{n}+T_{N}\right),\left(\sum_{n \in\{0,1, \cdots, N\}^{p}} \delta_{n} \cdot y_{n}+T_{N}\right)\right\rangle_{K_{N}} \\
& =\left\langle\left(\sum_{n \in\{0,1, \cdots, N\}^{p}} \delta_{n} \cdot x_{n}+T_{N}\right), A_{j}\left(\sum_{n \in\{0,1, \cdots, N\}^{p}} \delta_{n} \cdot y_{n}+T_{N}\right)\right\rangle_{K_{N}} \\
& =\left\langle\left(\sum_{n \in\{0,1, \cdots, N\}^{p}} \delta_{n} \cdot x_{n}+T_{N}\right), M_{j}\left(\sum_{n \in\{0,1, \cdots, N\}^{p}} \delta_{n} \cdot y_{n}^{\prime}+T_{N-1}\right)\right\rangle_{K_{N}} \\
& =\left\langle\left(\sum_{n \in\{0,1, \cdots, N\}^{p}} \delta_{n} \cdot x_{n}+T_{N}\right),\left(\sum_{n \in\{0,1, \cdots, N-1\}^{p}} \delta_{\left(n+e_{j}\right)} \cdot y_{n}^{\prime}+T_{N}\right)\right\rangle_{K_{N}} \\
& =\left\langle\left(\sum \delta_{n} x_{n}+T_{N}\right)+\left(\sum \delta_{n} \cdot x_{n}^{\prime}+T_{N-1}\right)-\left(\sum \delta_{n} \cdot x_{n}^{\prime}+T_{N-1}\right),\left(\sum \delta_{\left(n+e_{j}\right)} \cdot y_{n}^{\prime}+T_{N}\right)\right\rangle_{K_{N}} \\
& =\left\langle\left(\sum_{n \in\{0,1, \cdots, N-1\}^{p}} \delta_{n} \cdot x_{n}^{\prime}+T_{N}\right),\left(\sum_{n \in\{0,1, \cdots, N-1\}^{p}} \delta_{\left(n+e_{j}\right)} \cdot y_{n}^{\prime}+T_{N}\right)\right\rangle_{K_{N}} \\
& =\left\langle\sum_{n \in\{0,1, \cdots, N-1\}^{p}} h_{n} x_{n}^{\prime}, \sum_{n \in\{0,1, \cdots, N-1\}^{p}} h_{m+e_{j}} y_{n}^{\prime}\right\rangle_{K_{N}} \\
& =\left\langle\sum_{n, m \in\{0,1, \cdots, N-1\}^{p}} h_{\left(m+e_{j}\right)}^{*} h_{n} x_{n}^{\prime}, y_{m}^{\prime}\right\rangle_{\mathrm{H}} \\
& =\left\langle\sum_{n, m \in\{0,1, \cdots, N-1\}^{p}} \Gamma_{m+n+e_{j}} x_{n}^{\prime}, y_{m}^{\prime}\right\rangle_{\mathrm{H}}
\end{aligned}
$$

where we have denoted by $h_{n} x=\delta_{n} \cdot x+T_{N}$ (the class of $\delta_{n} \cdot x \in F_{N}$ with respect to $T_{N}$. The last statement is due to Kolmogorov's theorem of decomposition of positive kernels. 
We have also,

$$
\begin{aligned}
& \left\langle A_{j} \hat{x}, \hat{y}\right\rangle_{K_{N}}=\left\langle A_{j}\left(\sum_{n \in\{0,1, \cdots, N\}^{p}} \delta_{n} \cdot x_{n}+T_{N}\right),\left(\sum_{n \in\{0,1, \cdots, N\}^{p}} \delta_{n} \cdot y_{n}+T_{N}\right)\right\rangle_{K_{N}} \\
& =\left\langle M_{j}\left(\sum_{n \in\{0,1, \cdots, N-1\}^{p}} \delta_{n} \cdot x_{n}^{\prime}+T_{N-1}\right),\left(\sum_{n \in\{0,1, \cdots, N\}^{p}} \delta_{m} \cdot y_{m}+T_{N}\right)\right\rangle_{K_{N}} \\
& =\left\langle\left(\sum \delta_{\left(n+e_{j}\right)} \cdot x_{n}^{\prime}+T_{N}\right),\left(\sum \delta_{m} \cdot y_{m}-\sum \delta_{m} \cdot y_{m}^{\prime}+T_{N}+\sum \delta_{m} \cdot y_{m}^{\prime}+T_{N}\right)\right\rangle_{K_{N}} \\
& =\left\langle\left(\sum_{m \in\{0,1, \cdots, N-1\}^{p}} \delta_{\left(m+e_{j}\right)} \cdot x_{m}^{\prime}+T_{N}\right),\left(\sum_{m \in\{0,1, \cdots, N-1\}^{p}} \delta_{m} \cdot y_{m}^{\prime}+T_{N}\right)\right\rangle_{K_{N}} \\
& =\left\langle\sum_{m \in\{0,1, \cdots, N-1\}^{p}} h_{n+e_{j}} x_{n}^{\prime}, \sum_{m \in\{0,1, \cdots, N-1\}^{p}} h_{m} y_{m}^{\prime}\right\rangle_{K_{N}}=\left\langle\sum_{m \in\{0,1, \cdots, N-1\}^{p}} h_{m}^{*} h_{n+e_{j}} x_{n}^{\prime}, y_{m}^{\prime}\right\rangle_{\mathrm{H}} \\
& =\left\langle\sum \Gamma_{m+n+e_{j}} x^{\prime}, y_{m}^{\prime}\right\rangle_{\mathrm{H}} \text {; }
\end{aligned}
$$

From (8) and (9), it results that $\left\langle A_{j}^{*} \hat{x}, \hat{y}\right\rangle_{K_{N}}=$ $\left\langle A_{j} \hat{x}, \hat{y}\right\rangle_{K}$ for all $\hat{x}, \hat{y} \in K_{N}$ that is $A_{j}=A_{j}^{*}$.

Commutativity. We shall prove that $A_{k} \circ A_{j}=A_{j} \circ A_{k}$ for all $1 \leq j, k \leq p$. Following $A_{k}^{\prime}, A_{j}^{\prime}$ definitions, it is sufficient to verify in this order that

$M_{k} J_{N-1, N}^{-1} M_{j}=M_{j} J_{N-1, N}^{-1} M_{k}$ for all $1 \leq j, k \leq p$. Let $p \in F_{N-1}$ be the vectorial function $p=\sum_{l \in\{0,1, \ldots, N-1\}^{p}} \delta_{l} \cdot x_{l}$, we have:

$$
\begin{aligned}
M_{k}\left(p+T_{N-1}\right) & =M_{k}\left(\sum_{l \in\{0,1, \cdots, N-1\}^{p}} \delta_{l} \cdot x_{l}+T_{N-1}\right) \\
& =\sum_{l \in\{0,1, \cdots, N-1\}^{p}} \delta_{\left(l+e_{k}\right)} \cdot x_{l}+T_{N} .
\end{aligned}
$$

Because $\Lambda_{N}^{\Gamma}$ is stable at $(N-1), J_{N-1, N}$ is an isomorphism of vectorial spaces, it exists

$$
\begin{aligned}
& q_{j} \in F_{N-1}, q_{j}=\sum_{m \in\{0,1, \cdots, N-1\}^{p}} \delta_{m} \cdot y_{m}^{j} \text { such that: } \\
& \quad\left(q_{j}-M_{k} p\right)=\left(q_{j}-\sum_{l \in\{0,1, \cdots, N-1\}^{p}} \delta_{\left(l+e_{k}\right)} \cdot x_{l}\right) \in T_{N},
\end{aligned}
$$

case in which

$$
\begin{aligned}
& M_{j} J_{N-1, N}^{-1}\left(\sum_{l \in\{0,1, \cdots, N-1\}^{p}} \delta_{\left(l+e_{k}\right)} \cdot x_{l}+T_{N}\right) \\
& =M_{j}\left(q_{j}+T_{N-1}\right)=M_{j}\left(\sum_{m \in\{0,1, \cdots, N-1\}^{p}} \delta_{m} \cdot y_{m}^{j}+T_{N-1}\right) \\
& =\sum_{m \in\{0,1, \cdots, N-1\}^{p}} \delta_{\left(m+e_{j}\right)} \cdot y_{m}^{j}+T_{N}
\end{aligned}
$$

We compute also

$$
\begin{aligned}
& M_{k} J_{N-1, N}^{-1} M_{j}\left(p+T_{N-1}\right) \\
& =M_{k} J_{N-1, N}^{-1}\left(\sum_{l \in\{0,1, \cdots, N-1\}^{p}} \delta_{\left(l+e_{j}\right)} \cdot x_{l}+T_{N}\right) .
\end{aligned}
$$

Because $\Lambda_{N}^{\Gamma}$ is stable at $(N-1), J_{N-1, N}$ is an isomorphism, it exists $q_{k} \in F_{N-1}, q_{k}=\sum_{m \in\{0,1, \ldots, N-1\}^{p}} \delta_{m} \cdot z_{m}^{k}$ such that $\left(q_{k}-M_{j} p\right)=\left(q_{k}-\sum_{l \in\{0,1, \cdots, N-1\}^{p}} \delta_{l+e_{j}} \cdot x_{l}\right) \in T_{N}$ and

$$
\begin{aligned}
& M_{k} J_{N-1, N}^{-1}\left(\sum_{l \in\{0,1, \cdots, N-1\}^{p}} \delta_{l+e_{j}} \cdot x_{l}+T_{N}\right)=M_{k}\left(q_{k}+T_{N-1}\right) \\
& =M_{k}\left(\sum_{m \in\{0,1, \cdots, N-1\}^{p}} \delta_{m} \cdot z_{m}^{k}=\sum_{m \in\{0,1, \cdots, N-1\}^{p}} \delta_{m+e_{k}} \cdot z_{m}^{k}+T_{N}\right) .
\end{aligned}
$$

We prove that results in (10), (11) are equal; that is:

$$
\left(\sum_{m \in\{0,1, \cdots, N-1\}^{p}} \delta_{\left(m+e_{k}\right)} \cdot z_{m}^{k}-\sum_{m \in\{0,1, \cdots, N-1\}^{p}} \delta_{\left(m+e_{j}\right)} \cdot y_{m}^{j}\right) \in T_{N} .
$$

Indeed, let us consider the element

$$
f=\left(q_{j}-\sum_{l \in\{0,1, \cdots, N-1\}^{p}} \delta_{\left(l+e_{k}\right)} \cdot x_{l}\right) \in T_{N-1, N}^{1} \subset T_{N} .
$$

In these conditions, we can define $M_{j}\left(f+T_{N-1, N}^{1}\right) \in K_{N}$. Because $\Lambda_{N}^{\Gamma}$ is stable at $(N-1)$, we can find $r \in F_{N-1}$ such that $M_{j} f-r \in T_{N}$. In these conditions, from Cauchy-Buniakowski-Schwartz inequality and property b) in Remark 3.1, 


$$
\begin{aligned}
& \Lambda_{N}^{\Gamma}\left(M_{j} f, M_{j} f\right) \\
= & \Lambda_{N}^{\Gamma}\left(M_{j} f, r\right)+\Lambda_{N}^{\Gamma}\left(M_{j} f,\left(M_{j} f-r\right)\right) \\
= & \Lambda_{N}^{\Gamma}\left(f, M_{j} r\right)+\Lambda_{N}^{\Gamma}\left(M_{j} f,\left(M_{j} f-r\right)\right) \\
= & 0 \Rightarrow M_{j} f \in T_{N} \\
\Leftrightarrow & \left(\sum_{l \in\{0,1, \cdots, N-1\}^{p}} \delta_{\left(m+e_{j}\right)} \cdot y^{j}-\sum_{l \in\{0,1, \cdots, N-1\}^{p}} \delta_{\left(l+e_{j}+e_{k}\right)} \cdot x_{l}\right) \in T_{N} .
\end{aligned}
$$

Similarly, if we denote with

$$
g=\left(q_{k}-\sum_{l \in\{0,1, \cdots, N-1\}^{p}} \delta_{\left(l+e_{j}\right)} \cdot x_{l}\right) \in T_{N-1, N}^{1} \subset T_{N} ;
$$

we can define the extension

$$
\begin{aligned}
& M_{k}\left(g+T_{N-1 ; N}^{1}\right) \\
& =\sum_{l \in\{0,1, \cdots, N-1\}^{p}} \delta_{\left(m+e_{k}\right)} \cdot z_{m}^{k}-\left(\sum_{l \in\{0,1, \cdots, N-1\}^{p}} \delta_{\left(l+e_{j}+e_{k}\right)} \cdot x_{l}+T_{N}\right) .
\end{aligned}
$$

From the stability of $\Lambda_{N}^{\Gamma}$, respectively $J_{N-1, N}$ is an isomorphism, it exists $s \in F_{N-1}$ with $M_{k} g-s \in T_{N}$. From the same calculation as previous,

$$
\begin{aligned}
& \Lambda_{N}^{\Gamma}\left(M_{k} g, M_{k} g\right) \\
& =\Lambda_{N}^{\Gamma}\left(M_{k} g, s\right)+\Lambda_{N}^{\Gamma}\left(M_{k} g,\left(M_{k} g-s\right)\right) \\
& =\Lambda_{N}^{\Gamma}\left(g, M_{k} s\right)+\Lambda_{N}^{\Gamma}\left(M_{k} g,\left(M_{k} g-s\right)\right)=0 ;
\end{aligned}
$$

that is $M_{k} g \in T_{N}$. Consequently, we have:

$$
\begin{aligned}
M_{k} g & =M_{k}\left(q_{k}-\sum_{l} \delta_{\left(l+e_{j}\right)} \cdot x_{l}+T_{N-1, N}^{j}\right) \\
& =\sum_{m \in\{0,1, \cdots, N-1\}^{p}} \delta_{\left(m+e_{k}\right)} \cdot z_{m}^{k}-\sum \delta_{l+e_{j}+e_{k}} \cdot x_{l} \in T_{N} .
\end{aligned}
$$

From (12) and (13),

$$
\begin{aligned}
& \sum_{m \in\{0,1, \cdots, N-1\}^{p}} \delta_{m+e_{j}} \cdot y_{m}^{j}-\sum_{m \in\{0,1, \cdots, N-1\}^{p}} \delta_{m+e_{k}} \cdot z_{m}^{k} \\
= & \left(\sum_{m \in\{0,1, \cdots, N-1\}^{p}} \delta_{m+e_{j}} \cdot y_{m}^{j}-\sum_{l \in\{0,1, \cdots, N-1\}^{p}} \delta_{l+e_{j}+e_{k}} \cdot x_{l}\right) \\
+ & \left(\sum_{l \in\{0,1, \cdots, N-1\}^{p}} \delta_{l+e_{j}+e_{k}} \cdot x_{l}-\sum_{m \in\{0,1, \cdots, N-1\}^{p}} \delta_{m+e_{k}} \cdot z_{m}^{k}\right) \in T_{N} .
\end{aligned}
$$

That is results in (10) and (11) are equal; commutativity occurs.

Remark 4.4. In conditions of Proposition 4.3, we have $A^{m}\left[h_{0} x\right]=\left[h_{m} x\right]$ for all $m \in\{0,1, \cdots, N\}^{p}, \quad x \in \mathrm{H}$, where $\left[h_{0} x\right]$ stands for $\left[h_{0} x\right]=\widehat{\delta_{0} \cdot x}=\delta_{0} \cdot x+T_{N}$, respectively $\left[h_{m} x\right]=\widehat{\delta_{m} \cdot x}=\delta_{m} \cdot x+T_{N}, \delta_{0} \cdot x, \delta_{m} \cdot x \in F_{N}$ and $|m|=m_{1}+m_{2}+\cdots+m_{p}$.
Proof. Indeed, because $\Gamma_{N}$ is stable at $N-1$, if $|m| \leq p(N-1)$, from the definition of

$$
A=\left(A_{1}, \cdots, A_{p}\right), A_{i}: K_{N} \rightarrow K_{N},
$$

we have

$$
\begin{aligned}
A^{m}\left(\delta_{0} \cdot x+T_{N}\right) & =\prod_{i=1}^{p}\left(\left(M_{i} \circ J_{N-1, N}^{-1}\right)\left(\delta_{0} \cdot x+T_{N}\right)^{m_{i}}\right) \\
& =\delta_{m} \cdot x+T_{N}=\left[h_{m} x\right] .
\end{aligned}
$$

Let $\alpha \in\{0,1, \cdots, N\}^{p}$ with $p(N-1)<|\alpha| \leq p \cdot N$; we consider $\alpha=\beta+\gamma$ with $|\beta| \leq p(N-1)$ and $\gamma=\left(\gamma_{i}\right)_{i=1}^{p}$, $\gamma_{i}=1$ or $\gamma_{i}=0$. In this case, from Remark $4.2 \mathrm{~d}$ ) and first assertion of above,

$$
\begin{aligned}
A^{\alpha}\left[h_{0} x\right] & =\left(A^{\gamma} \circ A^{\beta}\right)\left(\left[h_{0} x\right]\right)=A^{\gamma}\left[h_{\beta} x\right]=A_{1}^{\gamma}\left[h_{\beta} x\right] \\
& =\left(J_{N-1, N}^{0,0+|\gamma|}\right)^{-1}\left(\delta_{\left(\beta+\gamma_{1} e_{1}+\cdots+\gamma_{p} e_{p}\right)}+T_{N-1, N}^{1 \cdots|\gamma|}\right) .
\end{aligned}
$$

From the definitions of the isomorphisms $\left(J_{N-1, N}^{k, k+1}\right)$, $\left(J_{N-1, N}^{0,0+|\gamma|}\right)$, it results

$$
A^{\alpha}\left[h_{0} x\right]-\left[\delta_{\left(\beta+\gamma_{1} e_{1}+\cdots+\gamma_{p} e_{p}\right)}\right]=0_{T_{N}} ;
$$

that is

$$
A^{\alpha}\left[h_{0} x\right]=\left[\delta_{\alpha} \cdot x+T_{N}\right]=\left[\delta_{\beta+\gamma} \cdot x+T_{N}\right]=\left[h_{\alpha} x\right]
$$

for all $\alpha \in\{0,1, \cdots, N\}^{p},|\alpha| \leq p \cdot N$.

Theorem 4.5. Let

$$
\begin{array}{r}
\Gamma_{N}=\left\{\left\{\Gamma_{m}\right\}_{m \in \mathrm{N}^{p}} \subset B(\mathrm{H}), n_{i} \leq 2 N,\right. \\
\left.1 \leq i \leq p, \Gamma_{n}=\Gamma_{n}^{*}, \Gamma_{0}=\operatorname{Id}_{\mathrm{H}}\right\}
\end{array}
$$

with the property $\sum_{m, n \in\{0,1, \cdots, N\}^{p}}\left\langle\Gamma_{n+m} x_{m}, x_{n}\right\rangle_{\mathrm{H}} \geq 0$ for any sequences $\left\{x_{n}\right\}_{n} \subset \mathrm{H}$. Let $F_{N}$ the vector space of vectorial functions and $\Lambda_{N}^{\Gamma}: F_{N} \times F_{N} \rightarrow \mathrm{C}$ the hspf associated with $\Gamma_{N}$, as in Remark 3.1, stable at $(N-1)$. Then there exists a unique extension $\Lambda_{\infty}: F_{\infty} \times F_{\infty} \rightarrow \mathrm{C}$ of $\Lambda_{N}^{\Gamma}$ which is a hspf on $F_{\infty} \times F_{\infty}$ and has property $b$ ) in Remark 3.1.

Proof. From Proposition 4.1, with the same notations as in Section 3, using the stability of $\Lambda_{N}^{\Gamma}$ at $(N-1)$, we can define a $p$ commuting tuple of selfadjoint operators $A=\left(A_{1}, \cdots, A_{p}\right), A_{i}: K_{N} \rightarrow K_{N}, 1 \leq i \leq p$. For an arbitrary $l \geq N$ and $p \in F_{l}, p=\sum_{k \in\{0,1, \cdots, l\}^{p}} \delta_{k} p(k)$, we define the element

$$
\begin{aligned}
& p(A)=\sum_{k \in\{0,1, \cdots, l\}^{p}} A^{k}\left[h_{0} p(k)\right] \in K_{N}, \\
& \text { with }\left[h_{0} p(k)\right]=\delta_{0} p(k)+T_{N},
\end{aligned}
$$

when $A^{k}=A_{1}^{k_{1}} \circ \cdots \circ A_{p}^{k_{p}}, k=\left(k_{1}, \cdots, k_{p}\right)$. Let us consider the functional $\Lambda_{\infty}: F_{\infty} \times F_{\infty} \rightarrow \mathrm{C}$ defined by $\Lambda_{\infty}(p, q)=$ 
$\langle p(A), q(A)\rangle_{K_{N}}$ when $p, q \in F_{l}$ with $l \geq N$ arbitrary.

We prove in the sequel that $\Lambda_{\infty}: F_{\infty} \times F_{\infty} \rightarrow \mathrm{C}$ is an extension of $\Lambda_{N}^{\Gamma}: F_{N} \times F_{N} \rightarrow C$, it is a hspf on $F_{\infty} \times F_{\infty}$ and it has property b) in Remark 3.1.

From the properties of the scalar product on $K_{N}$ and definition of $p(A), q(A) \in K_{N}$ above, we have

$$
\Lambda_{\infty}\left(\alpha_{1} p_{1}+\alpha_{2} p_{2}, h\right)=\alpha_{1} \Lambda_{\infty}\left(p_{1}, h\right)+\alpha_{2} \Lambda_{\infty}\left(p_{2}, h\right)
$$

for all $\alpha_{1}, \alpha_{2} \in \mathrm{C}, p_{1}, p_{2} \in F_{l}, l \geq N$ arbitrary. Obviously, using the same properties, we have:

$$
\begin{gathered}
\overline{\Lambda_{\infty}(f, g)}=\Lambda_{\infty}(g, f) \text { and } \Lambda_{\infty}(f, f)=\|f(A)\|_{K_{N}}^{2} \geq 0, \\
l \geq N, \quad f, g \in F_{l}
\end{gathered}
$$

arbitrary. It results that $\Lambda_{\infty}: F_{\infty} \times F_{\infty} \rightarrow \mathrm{C}$ is a hspf on $F_{\infty} \times F_{\infty}$.

We verify that $\Lambda_{\infty}: F_{\infty} \times F_{\infty} \rightarrow \mathrm{C}$ is an extension of $\Lambda_{N}^{\Gamma}: F_{N} \times F_{N} \rightarrow \mathrm{C}$. For any vector-valued functions

$$
p, q \in F_{N}, p=\sum_{k \in\{0,1, \cdots, N\}^{p}} \delta_{k} \cdot p(k), q=\sum_{j \in\{0,1, \cdots, N\}^{p}} \delta_{j} \cdot q(i),
$$

we have:

$$
\begin{aligned}
& \Lambda_{\infty}(p, q) \\
& =\left\langle\sum_{k \in\{0,1, \cdots, N\}^{p}} A^{k}\left[h_{0} p(k)\right], \sum_{i \in\{0,1, \cdots, N\}^{p}} A^{i}\left[h_{0} p(i)\right]\right\rangle_{K_{N}} \\
& =\left\langle\sum_{k \in\{0,1, \cdots, N\}^{p}}\left[h_{k} p(k)\right], \sum_{i \in\{0,1, \cdots, N\}^{p}}\left[h_{i} p(i)\right]\right\rangle_{K_{N}} \\
& =\sum_{i, k \in\{0,1, \cdots, N\}^{p}}\left\langle h_{i}^{*} h_{k} p(k), q(i)\right\rangle_{\mathrm{H}} \\
& =\sum_{i, k \in\{0,1, \cdots, N\}^{p}}\langle\Gamma(i, k) p(k), q(i)\rangle_{\mathrm{H}} \\
& =\sum_{i, k \in\{0,1, \cdots, N\}^{p}}\left\langle\Gamma_{i+k} p(k), q(i)\right\rangle_{\mathrm{H}}=\Lambda_{N}^{\Gamma}(p, q) ;
\end{aligned}
$$

that is $\Lambda_{\infty}$ is an extension as hspf to $F_{\infty} \times F_{\infty}$ of $\Lambda_{N}^{\Gamma}$ (the above results uses Remark 4.4 and Kolmogorov's theorem).

We verify that $\Lambda_{\infty}$ has also property b) in Remark 3.1 , respectively it fullfiels

$$
\Lambda_{\infty}\left(p * \delta_{e_{i}}, q\right)=\Lambda_{\infty}\left(p, q * \delta_{e_{i}}\right),
$$

for all $1 \leq i \leq p, p, q \in F_{l}, l \geq N$, arbitrary. Indeed,

$$
\begin{aligned}
& \Lambda_{\infty}\left(p * \delta_{e_{i}}, q\right) \\
& =\left\langle\sum_{k \in\{0,1, \cdots, l\}^{p}} A^{k+e_{i}}\left(\left[h_{0} p(k)\right]\right), \sum_{j \in\{0,1, \cdots, l\}^{p}} A^{j}\left(\left[h_{0} q(j)\right]\right)\right\rangle_{K_{N}} \\
& =\left\langle\sum_{k \in\{0,1, \cdots, l\}^{p}} A^{k}\left(\left[h_{0} p(k)\right]\right), \sum_{i \in\{0,1, \cdots, l\}^{p}} A^{j+e_{i}}\left(\left[h_{0} q(j)\right]\right)\right\rangle_{K_{N}} \\
& =\Lambda_{\infty}\left(p, q * \delta_{e_{i}}\right)
\end{aligned}
$$

the required property. We prove that $\Lambda_{\infty}$ is the unique extension of $\Lambda_{N}^{\Gamma}$ with the mentioned properties. Suppose that $\Lambda^{\prime}, \Lambda^{\prime \prime}$ are two extensions to $F_{\infty} \times F_{\infty}$ of $\Lambda_{N}^{\Gamma}$, both of them with the specified properties. We prove by recurrence, that for every $l, l \geq N$, and any vectorial function $\delta_{l} \cdot x_{l} \in F_{l}$, there exists $p \in F_{N-1}$ such that $\left(\delta_{l_{1}} \cdot x_{l}-p\right) \in T_{l}^{\prime} \cap T_{l}^{\prime \prime}$. For $l=N$ and $\delta_{N} x \in F_{N}$, because $J_{N-1, N}$ is an isomorphism, exists then an element $p \in F_{N-1}$ such that $\left(\delta_{(N, \cdots, N)} \cdot x-p\right) \in T_{N}=T_{N}^{\prime}=T_{N}^{\prime \prime}$; that is the required assertion, in case $l=N$, is true. We consider the statement satisfied in case $(l+k-1) \geq N$ and prove it for $(l-k) \geq N$; that is for any $\delta_{l_{1}+k_{1}-1} \cdot x \in F_{l+k-1}$, with $l_{1}=(l, \cdots, l), k_{1}=(k, \cdots, k) \in \mathrm{N}^{p}$, there is an element $p \in F_{N-1}, \quad p=\sum_{k_{1} \in\{0,1, \cdots, N-1\}^{p}} \delta_{k_{1}} \cdot p(k)$ such that $\left(\delta_{l_{1}+k_{1}-1} x-p\right) \in T_{l_{1}+k_{1}-1}^{\prime} \cap T_{l_{1}+k_{1}-1}^{\prime \prime}$. This relation means that

$$
\begin{aligned}
& \Lambda_{l_{1}+k_{1}-1}^{\prime} \cdot\left(\left(\delta_{l_{1}+k_{1}-1} \cdot x-\sum_{k_{1} \in\{0,1, \cdots, N-1\}^{p}} \delta_{k_{1}} \cdot p\left(k_{1}\right)\right), \delta_{l_{1}+k_{1}-1} \cdot x-\sum_{k \in\{0,1, \cdots, N-1\}^{p}} \delta_{k} \cdot p(k)\right) \\
& =\Lambda_{l_{1}+k_{1}-1}^{\prime \prime}\left(\left(\delta_{l_{1}+k_{1}-1} \cdot x-\sum_{k_{1} \in\{0,1, \cdots, N-1\}^{p}} \delta_{k_{1}} \cdot p\left(k_{1}\right)\right), \delta_{l_{1}+k_{1}-1} \cdot x-\sum_{k \in\{0,1, \cdots, N-1\}^{p}} \delta_{k} \cdot p(k)\right)=0 .
\end{aligned}
$$

We compute:

$$
\begin{aligned}
& \left|\Lambda_{l_{1}+k_{1}}^{\prime}\left(\delta_{l_{1}+k_{1}} \cdot x-\sum_{k_{1} \in\{0,1, \cdots, N-1\}^{p}} \delta_{k_{1}+1} \cdot p\left(k_{1}\right), \delta_{l_{1}+k_{1}} \cdot x-\sum_{k_{1} \in\{0,1, \cdots, N-1\}^{p}} \delta_{k_{1}+1} \cdot p(k)\right)\right| \\
& =\left|\Lambda_{l_{1}+k_{1}-1}^{\prime}\left(\delta_{l_{1}+k_{1}-1} \cdot x-\sum_{k_{1} \in\{0,1, \cdots, N-1\}^{p}} \delta_{k_{1}} \cdot p\left(k_{1}\right)\right), \delta_{l_{1}+k_{1}+1} \cdot x-\sum_{k_{1} \in\{0,1, \cdots, N-1\}^{p}} \delta_{k_{1}+2} \cdot p\left(k_{1}\right)\right| \\
& \leq \Lambda^{\prime}\left(\delta_{l_{1}+k_{1}-1} X-\sum \delta_{k_{1}} \cdot p\left(k_{1}\right), \delta_{l_{1}+k_{1}-1} \cdot x-\sum \delta_{k_{1}} \cdot p\left(k_{1}\right)\right)^{\frac{1}{2}} \Lambda^{\prime}(h, h)^{\frac{1}{2}}=0 ;
\end{aligned}
$$


where we have denoted with:

$$
h=\delta_{l_{1}+k_{1}-1} \cdot x-\sum_{k_{1} \in\{0,1, \cdots, N-1\}^{p}} \delta_{k_{1}+2} \cdot p(k) .
$$

The same inequality is true for $\Lambda^{\prime \prime}$; it results that

$$
\delta_{l_{1}+k_{1}} x-\sum_{k_{1} \in\{0,1, \cdots, N-1\}^{p}} \delta_{k_{1}+1} \cdot p\left(k_{1}\right) \in T_{l+k}^{\prime} \cap T_{l+k}^{\prime \prime} .
$$

The vectorial function

$$
\sum_{k_{1} \in\{0,1, \cdots, N-1\}^{p}} \delta_{k_{1}+1} \cdot p\left(k_{1}\right) \in F_{N}, \quad J_{N-1, N}
$$

is an isomorphism $\left(\Gamma_{N}\right.$ is stable at $\left.N-1\right)$, there exists $p \in F_{N-1}$ such that

$$
\begin{aligned}
& \Lambda_{N}^{\Gamma}\left(p-\delta_{k_{1}+1} \cdot p\left(k_{1}\right), p-\sum \delta_{k_{1}+1} \cdot p\left(k_{1}\right)\right) \\
& =\Lambda^{\prime}\left(p-\delta_{k_{1}+1} \cdot p\left(k_{1}\right), p-\sum \delta_{k_{1}+1} \cdot p\left(k_{1}\right)\right) \\
& =\Lambda^{\prime \prime}\left(p-\delta_{k_{1}+1} \cdot p\left(k_{1}\right), p-\sum \delta_{k_{1}+1} \cdot p\left(k_{1}\right)\right)=0 ;
\end{aligned}
$$

that is $\left(p-\sum_{k_{1} \in\{0,1, \cdots, N-1\}^{p}} \delta_{k_{1}+1} \cdot p\left(k_{1}\right)\right) \in T_{N} \cap T_{l+k}^{\prime} \cap T_{l+k}^{\prime \prime}$.

We have:

$$
\begin{aligned}
& \delta_{l_{1}+k_{1}} \cdot x-p \\
& =\left(\delta_{l_{1}+k_{1}} \cdot x-\sum_{k_{1} \in\{0,1, \cdots, N-1\}^{p}} \delta_{k_{1}+1} \cdot p\left(k_{1}\right)\right) \\
& +\left(\sum_{k_{1} \in\{0,1, \cdots, N-1\}^{p}} \delta_{k_{1}+1} \cdot p\left(k_{1}\right)-p\right) \in T_{l+k}^{\prime} \cap T_{l+k}^{\prime \prime} .
\end{aligned}
$$

For every $l \geq 0$, and any $p, q \in F_{N+l}$, it results, from above, that we can find the elements $p_{1}, p_{2} \in F_{N-1}$ such that $p-p_{1}, q-p_{2} \in T_{N+1}^{\prime} \cap T_{N+1}^{\prime \prime}$. Moreover,

$$
\begin{aligned}
\Lambda^{\prime}(p, q) & =\left\langle p+T_{N+l}^{\prime}, q+T_{N+1}^{\prime}\right\rangle K_{N+l}^{\prime} \\
& =\left\langle p_{1}+T_{N}, p_{2}+T_{N}\right\rangle K_{N} \\
& =\left\langle p+T_{N+l}^{\prime \prime}, q+T_{N+1}^{\prime \prime}\right\rangle_{K_{N+1}}=\Lambda_{N}^{\Gamma}(p, q) ;
\end{aligned}
$$

showing that $\Lambda^{\prime}\left|F_{N+1} \times F_{N+1}=\Lambda^{\prime \prime}\right| F_{N+1} \times F_{N+l}$. The integer $l \geq 0$ is arbitrary choosen, we obtain that

$\Lambda^{\prime} \mid F_{\infty} \times F_{\infty=\Lambda^{\prime \prime} \mid F_{\infty} \times F_{\infty}}$; that is the extension with such properties is unique.

Remark 4.6. Let $\Lambda_{\infty}: F_{\infty} \times F_{\infty} \rightarrow \mathrm{C}$ be defined by $\Lambda_{\infty}(p, q)=\langle p(A), q(A)\rangle_{K_{N}}$, for any $p, q \in F_{l}, l \geq N$. In this case, the null space is

$$
T_{l}=\left\{f \in F_{l}, f=\sum_{k \in\{0,1, \cdots,\}^{p}} \delta_{k} \cdot f(k) \in F_{l}, \Lambda_{\infty}(f, f)=\|f(A)\|_{K_{N}}^{2}=0\right\} .
$$

It results that, for any $f \in T_{l}$ there exists $p \in T_{N}$ such that $p(A)=f(A)$; we prove that $T_{l+1} \subset T_{l+2}$ for any $l \geq N-1$.

Let $f=\sum_{k \in\{0,1, \ldots, l+1\}^{p}} \delta_{k} \cdot f(k) \in F_{l+1}$ such that

$$
\begin{gathered}
\Lambda_{\infty} \mid F_{l+1} \times F_{l+1}(f, f)=\langle f(A), f(A)\rangle_{K_{N}}=\left\langle\sum_{k \in\{0,1, \cdots, l+1\}^{p}} A^{k}\left[h_{0} f(k)\right], \sum_{k \in\{0,1, \cdots, l+1\}^{p}} A^{k}\left[h_{0} f(k)\right]\right\rangle_{K_{N}}=0 . \\
J_{l+1, l+2}(f)=\sum_{k \in\{0,1, \cdots, l+1\}^{p}} \delta_{k} \cdot f(k)+\delta_{l+2} 0_{H}+T_{l+2}, \text { it follows } \\
\Lambda_{\infty} \mid F_{l+2} \times F_{l+2}(f, f)=\left\langle\sum_{k \in\{0,1, \cdots, l+1\}^{p}} A^{k}\left[h_{0} f(k)\right], \sum_{k \in\{0,1, \cdots, l+1\}^{p}} A^{k}\left[h_{0} f(k)\right]\right\rangle_{K_{N}}=0,
\end{gathered}
$$

that is $T_{l+1} \subset T_{l+2}$ for any $l \geq N$; consequently, $K_{l+1} \subset K_{l+2}$ for any $l \geq N-1$.

Proposition 4.7. Let $\Gamma_{N}=\left\{\Gamma_{n}\right\}_{n \in \mathbb{N}^{p}, n_{i} \leq 2 N} \subset B(\mathrm{H})$ be an operator kernel, positively defined and $\Lambda_{N}^{\Gamma}: F_{N} \times F_{N} \rightarrow \mathrm{C}$ the hspf associated with the kernel $\Gamma_{N}$ as previous, stable at $(N-1)$ and

$\Lambda_{\infty}: F_{\infty} \times F_{\infty} \rightarrow \mathrm{C}$, the unique extension of $\Lambda_{N}^{\Gamma}$ to $F_{\infty} \times F_{\infty}$ as hspf and property b) in Remark 3.1. Then $\Lambda_{\infty}$ is stable at any $l \geq N-1$.

Proof. Let $\left\{K_{l}\right\}_{l>0}$ be the Hilbert spaces built via $\Lambda_{\infty}$ and $J_{l, l+1}: K_{l} \rightarrow K_{l+1}$ be the associated isometries. We prove, by induction, that $J_{l, l+1}\left(K_{l}\right)=K_{l+1}$ for all $l \geq N-1$. The assertion is true for $l=N \quad\left(J_{N-1, N}\right.$ is an isomorthism, $\Lambda_{N}^{\Gamma}=\Lambda_{\infty} \mid F_{N} \times F_{N}$ is stable at $N$ ). Assume that the assertion is true for some $l \geq N$ and prove it for $(l+1)$. We fix an element $\delta_{l+2} x \in F_{l+2}$ and prove that, we can find an element $p_{l+1}=\sum_{k \in\{0,1, \cdots, l+1\}^{p}} \delta_{k} \cdot x(k) \in F_{l+1}$ such that $\delta_{l+1} X-p_{l+1} \in T_{l+2}$, with $T_{l+2}$ the null space of $\left.\Lambda_{\infty}\right|_{F_{l+2} \times F_{l+2}}$. For the element $\delta_{l+1} x \in F_{l+1}$, because $J_{l, l+1}$ is an isomorphism, it exists then

$p=\sum_{k \in\{0,1, \cdots,\}^{p}} \delta_{k} \cdot p(k) \in F_{l}$ such that

$\delta_{l+1} x-\sum_{k \in\{0,1, \cdots, l\}^{p}} \delta_{k} \cdot p(k) \in T_{l+1}$, that is 


$$
\Lambda_{\infty}\left(\delta_{l+1} x-\sum_{k \in\{0,1, \cdots, l\}^{p}} \delta_{k} \cdot p(k), \delta_{l+1} \cdot x-\sum_{k \in\{0,1, \cdots, l\}^{p}} \delta_{k} \cdot p(k)\right)=0 .
$$

Using the property b) in Remark 3.1 for $\Lambda_{\infty}$, we compute:

$$
\begin{aligned}
& \left|\Lambda_{\infty}\left(\delta_{l+2} \cdot x-\sum_{k \in\{0,1, \cdots, l\}^{p}} \delta_{k+1} \cdot p(k), \delta_{l+2} x-\sum_{k \in\{0,1, \cdots, l\}^{p}} \delta_{k+1} \cdot p(k)\right)\right| \\
& =\left|\Lambda_{\infty}\left(\delta_{l+1} \cdot x-\sum_{k \in\{0,1, \cdots, l\}^{p}} \delta_{k} \cdot p(k), \delta_{l+3} \cdot x-\sum_{k \in\{0,1, \cdots, l\}^{p}} \delta_{k} \cdot p(k)\right)\right| \\
& \leq \Lambda_{\infty}\left(\delta_{l+1} \cdot x-\sum \delta_{k} p(k)\right), \delta_{l+1} \cdot x-\sum \delta_{k} \cdot p(k)^{\frac{1}{2}}\left(\Lambda_{\infty}(g, g)\right)^{\frac{1}{2}}=0 ;
\end{aligned}
$$

where we have denoted with

$$
g=\left(\delta_{l+3} \cdot x-\sum_{k \in\{0,1, \ldots,\}^{p}} \delta_{k+2} \cdot p(k)\right) .
$$

That is $J_{l+1, l+2}: K_{l+1} \rightarrow K_{l+2}$ is a surjective isometry, insures that $J_{l+1, l+2}$ is an isomorphism of vectorial spaces. By recurrence, $\Lambda_{\infty}$, the unique extension of $\Lambda_{N}^{\Gamma}$ as a hspf with property b) in Remark 3.1 is stable at any $l \geq N-1$.

Corollary 4.8. Let $\Gamma_{N}$ be an operator-valued kernel, positively defined and $\Lambda_{N}^{\Gamma}: F_{N} \times F_{N} \rightarrow \mathrm{C}$ the hspf associated with $\Gamma_{N}$ as above. If $\Lambda_{N}^{\Gamma}: F_{N} \times F_{N} \rightarrow \mathrm{C}$ is stable at one indices $k \in\{0,1, \cdots, N-1\}$, then $\Lambda_{N}^{\Gamma}$ is stable at any $l \geq k, l \in\{k, k+1, \cdots, N-1\}$.

Proof. The unique extension of $\Lambda_{N}^{\Gamma} \mid F_{k+1} \times F_{k+1} \rightarrow \mathrm{C}$, $k+1 \leq N$ as a hspf and with property b) in Remark 3.1 is the $\Lambda_{\infty}$ extension which is stable at any $l \geq k$ that is $\Lambda_{N}^{\Gamma}\left|F_{k+1} \times F_{k+1}=\Lambda_{\infty}\right| F_{k+1} \times F_{k+1}$ is stable at $(k+1)$. It follows by recurrence that $\Lambda_{N}^{\Gamma}$ is stable at any $l \in\{k, k+1, \cdots, N-1\}$.

In the sequel, we argue like in [1], Remark 2.9.

Remark 4.9. Let $\Gamma_{N}=\left\{\Gamma_{n}\right\}_{n \in \mathbb{N}^{p}, n_{i} \leq 2 N} \subset B(\mathrm{H})$ be an operator kernel, positively defined, $\Lambda_{N}^{n_{i}}: F_{N} \times F_{N} \rightarrow \mathrm{C}$ the hspf associated with the kernel $\Gamma_{N}$ as previous, stable at $(N-1)$ and $\Lambda_{\infty}: F_{\infty} \times F_{\infty} \rightarrow \mathrm{C}$, the unique extension of $\Lambda_{N}^{\Gamma}$ to $F_{\infty} \times F_{\infty}$ as hspf and with property b) in Remark 3.1, defined in Proposition 4.5. Let $\left\{K_{l}\right\}_{l \geq N}$ be the Hilbert spaces constructed via $\Lambda_{\infty}$; because $\Lambda_{\infty}$ is stable at any $k \geq N-1$, the isometries $J_{l, j}: K_{l} \rightarrow K_{j}, \quad j \geq l, \quad J_{l, j}=J_{l, l+1} \circ J_{l+1, l+2} \circ \cdots \circ J_{j-1, j}$ are bijective one, that is $J_{l, j}\left(K_{l}\right)=K_{j}$. We denote with $J_{l, l+1}=J_{l}$. We may construct the $p$-tuple of commuting selfadjoint operators $A_{l}=\left(A_{l 1}, \cdots, A_{l p}\right)$ on the space $K_{l}$, $l \geq N$, as in Proposition 4.2. We define as in Remark 4.1

(A) the operators $M_{l-1 j}: K_{l-1} \rightarrow K_{l}$ by

$$
\begin{aligned}
& M_{l-1 j}\left(p+T_{l-1}\right)=M_{l-1 j}\left(\sum_{n \in\{0,1, \cdots, l-1\}^{p}} \delta_{n} \cdot p(n)+T_{l-1}\right) \\
& =\left(\sum_{n \in\{0,1, \cdots, l-1\}^{p}} \delta_{n+e_{j}} \cdot p(n)+T_{l}\right)=\sum_{n \in\{0,1, \cdots, l-1\}^{p}}\left[h_{n} p(n)\right] .
\end{aligned}
$$

We have immediately:

$$
\begin{aligned}
& M_{l j} J_{l-1}\left(\sum_{n \in\{0,1, \cdots, l-1\}^{p}} \delta_{n} \cdot p(n)+T_{l-1}\right) \\
& =M_{l j}\left(\sum_{n \in\{0,1, \cdots, l-1\}^{p}} \delta_{n} \cdot p(n)+T_{l}\right) \\
& =\left(\sum_{n \in\{0,1, \cdots, l-1\}^{p}} \delta_{n} \cdot p(n)+T_{l+1}\right) \\
& =\sum_{n \in\{0,1, \cdots, l-1\}^{p}}\left[h_{n} p(n)\right] .
\end{aligned}
$$

modulo $T_{l+1}$ for all $l \geq N$. It results $J_{l} M_{l-1 j}=M_{l j} J_{l-1}$ that imply $M_{l k} M_{l-1 j}=M_{l k} J_{l}^{-1} M_{l j} J_{l-1}$; respectively

$$
\begin{aligned}
& M_{l k} M_{l-1 j}\left(\sum_{n i n \in\{0,1, \cdots, l-1\}^{p}} \delta_{n} \cdot p(n)+T_{l-1}\right) \\
& =\sum_{n \in\{0,1, \cdots, l-1\}^{p}} \delta_{n+e_{j}+e_{j}} \cdot p(n)+T_{l+1} .
\end{aligned}
$$

Consequently

$$
\begin{aligned}
& A_{l k} A_{l j}\left(\sum_{n \in\{0,1, \cdots, l-1\}^{p}} \delta_{n} \cdot p(n)+T_{l-1}\right) \\
& =A_{l k} M_{l-1 j} J_{l-1}^{-1}\left(p+T_{l-1}\right)=M_{l-1 k} J_{l-1}^{-1} J_{l}^{-1} M_{l j} \\
& =J_{l}^{-1}\left(M_{l k} J_{l}^{-1}\right) M_{l j}=J_{l}^{-1} J_{l+1}^{-1} M_{l+1} M_{l j} \\
& =J_{l(l+2)}^{-1} M_{l+1} M_{l j} ;
\end{aligned}
$$

consequently we obtain:

$$
\begin{aligned}
& A_{l j} A_{l k}\left(\sum_{n \in\{0,1, \cdots, l-1\}^{p}} \delta_{n} \cdot p(n)+T_{l}\right) \\
& =J_{l, l+2}^{-1}\left(\sum_{n \in\{0,1, \cdots, l-1\}^{p}} \delta_{n+e_{j}+e_{k}} \cdot p(n)+T_{l+2}\right) .
\end{aligned}
$$

A recurrence argument leads to the formula: 


$$
\begin{aligned}
& A_{l}^{\alpha}\left(\sum_{n \in\{0,1, \cdots, l-1\}^{p}} \delta_{n} \cdot p(n)+T_{l}\right) \\
& =J_{l(l+k)}^{-1}\left(\sum_{n \in\{0,1, \cdots, l-1\}^{p}} \delta_{n+\alpha_{1} e_{1}+\cdots+\alpha_{p} e_{p}} \cdot p(n)+T_{l+k}\right), \\
& |\alpha|=k .
\end{aligned}
$$

Let

$$
\begin{aligned}
\Gamma_{N} & =\left\{\Gamma_{n} \in B(\mathrm{H}), n \in \mathrm{N}^{p}, n_{i} \leq 2 N, 1 \leq i \leq p\right\} \\
& =\left\{\Gamma_{p+q} \in B(\mathrm{H}), p, q \in \mathrm{N}^{p}, p_{i}, q_{i} \leq N, 1 \leq i \leq p\right\},
\end{aligned}
$$

subject on the same conditions as in Remark 4.2. We denote with $a_{p+q}^{i j}=\left\langle\Gamma_{p+q} v_{i}, v_{j}\right\rangle_{\mathrm{H}}$ with $v_{i}, v_{j}$ C-linear vectors in a basis of $\mathrm{H}, 1 \leq i, j \leq \operatorname{dim}_{\mathrm{C}} \mathrm{H}$.

Proposition 4.10. Let $\Gamma_{N}$ be an operator kernel, stable at $N-1$, as above. We consider that:

1) for all $\Gamma_{2 n}, n_{i}<N, \forall 1 \leq i \leq p$, we have $a_{i i}^{2 n} a_{j j}^{2 n}-a_{i j}^{2 n} a_{j i}^{2 n} \neq 0$, with $v_{i} \neq v_{j}, v_{i}, v_{j}$ vectors in a basis of $\mathrm{H}$, and $a_{i j}^{2 n}=\left\langle\Gamma_{2 n} v_{i}, v_{j}\right\rangle_{\mathrm{H}}$ for all $1 \leq i$, $j \leq \operatorname{dim} \mathrm{H}$. In this case, for all $n \in \mathrm{N}^{p}, n_{i}<N, 1 \leq i \leq p$, the elements $\delta_{n} \cdot v_{i}, \delta_{n} \cdot v_{j} \in F_{N-1}$ are linear independent in $K_{N-1}$.

2) Moreover, we consider in addition, that the kernel $\Gamma_{N}=\left\{\Gamma_{n} \in B(\mathrm{H}), n \in \mathrm{N}^{p}, n_{i} \leq 2 N, 1 \leq i \leq p\right\}$ is such that for all $v_{i}$, as in 1$), 1 \leq i \leq \operatorname{dim}_{\mathrm{C}} \mathrm{H}$ the elements

$$
\delta_{0} \cdot v_{i}+T_{N-1}, \delta_{e_{k}} \cdot v_{i}+T_{N-1}, \quad 1 \leq k \leq p, \cdots, \delta_{N-1} \cdot v_{i}+T_{N-1} .
$$

are C-linear independent in $K_{N-1}$ and for any $v_{i}$, $1 \leq i \leq \operatorname{dim}_{\mathrm{C}} \mathrm{H}$, and any $1 \leq k \leq p$ the elements

$$
\begin{aligned}
& \delta_{0} \cdot v_{i}+T_{N}, \delta_{e_{1}} \cdot v_{i}+T_{N}, \cdots, \delta_{(N-1)} \cdot v_{i} \\
& +T_{N}, \delta_{(N-1)+e_{k}} \cdot v_{i}+T_{N}
\end{aligned}
$$

are linear dependent in $K_{N}$ (in $\delta_{(N-1)+e_{k}},(N-1)$ stands for $\left.(N-1, N-1, \cdots, N-1) \in \mathrm{N}^{p}\right)$.

Proof. 1) Indeed, let us show, that, if we have $\alpha, \beta \in \mathrm{C}$ such that (14)

$$
\Lambda_{N}^{\Gamma}\left\langle\left(\alpha \delta_{n} \cdot v_{i}+\beta \delta_{n} \cdot v_{j}\right), \alpha \delta_{n} \cdot v_{i}+\beta \delta_{n} \cdot v_{j}\right\rangle_{\mathrm{H}}=0
$$

it results $\alpha=\beta=0$. We have

$$
\begin{aligned}
& \Leftrightarrow \sum_{l, m \in\{0,1, \cdots, N\}^{p}}\left\langle\Gamma_{l, m}\left(\alpha \delta_{n} \cdot v_{i}+\beta \delta_{n} \cdot v_{j}\right), \alpha \delta_{n} \cdot v_{i}+\beta \delta_{n} \cdot v_{j}\right\rangle_{\mathrm{H}}=0 \\
& \Leftrightarrow\left\langle\Gamma(n+n)\left(\alpha \delta_{n} \cdot v_{i}+\beta \delta_{n} \cdot v_{j}\right), \alpha \delta_{n} v_{i}+\beta \delta_{n} \cdot v_{j}\right\rangle_{\mathrm{H}}=0 \\
& \Leftrightarrow|\alpha|^{2} a_{i i}^{2 n}+|\beta|^{2} a_{j j}^{2 n}+\alpha \bar{\beta} a_{i j}^{2 n}+\beta \bar{\alpha} a_{j i}^{2 n}=0 .
\end{aligned}
$$

Immediately, from above, we have:

$$
\left(|\alpha|\left(a_{i i}^{2 n}\right)^{\frac{1}{2}}-|\beta|\left(a_{j j}^{2 n}\right)^{\frac{1}{2}}\right)^{2}+2|\alpha||\beta|\left(a_{i i}^{2 n} a_{j j}^{2 n}\right)^{\frac{1}{2}}+\alpha \bar{\beta} a_{i j}^{2 n}+\beta \bar{\alpha} a_{j i}^{2 n}=0 .
$$

In the same time, from Cauchy-Buniakowski-Schwarz inequality, we have:

$$
\begin{aligned}
\left|\Lambda_{N}^{\Gamma}\left(\alpha \delta_{n} \cdot v_{i}, \beta \delta_{n} \cdot v_{j}\right)\right| & \leq \Lambda_{N}^{\Gamma}\left(\alpha \delta_{n} \cdot v_{i}, \alpha \delta_{n} \cdot v_{j}\right)^{\frac{1}{2}} \Lambda_{N}^{\Gamma}\left(\beta \delta_{n} \cdot v_{i}, \beta \delta_{n} \cdot v_{j}\right)^{\frac{1}{2}} \\
& \Leftrightarrow|\alpha||\beta|\left|a_{i j}^{2 n}\right| \leq|\alpha||\beta|\left(a_{i i}^{2 n}\right)^{\frac{1}{2}}\left(a_{j j}^{2 n}\right)^{\frac{1}{2}} .
\end{aligned}
$$

Equatities (15) can be satisfied, in case of (16), only when

$$
\left\{\begin{array}{l}
|\alpha|^{2} a_{i i}^{2 n}=|\beta|^{2} a_{j j}^{2 n} \\
-\alpha \bar{\beta} a_{i j}^{2 n}=|\alpha||\beta|\left|a_{i j}^{2 n}\right| \\
-\beta \bar{\alpha} a_{j i}^{2 n}=|\alpha||\beta|\left|a_{j i}^{2 n}\right|
\end{array}\right.
$$

are true. In condition of Proposition 4.10. 1), the only case in which (17) can happen is $\alpha=\beta=0$; that is $\delta_{n} \cdot v_{i}, \delta_{n} \cdot v_{j}$ are linear independent elements in $K_{N-1}$.

Theorem 4.11. Let $\Gamma_{N}=\left\{\Gamma_{n}\right\}_{n \in \mathbb{N}^{p}, n_{i} \leq 2 N} \subset B(\mathrm{H})$ be an operator kernel, positively defined, stable at $N-1$, such that its terms $\Gamma_{n}$ satisfy conditions 1) and 2) in Proposition 4.10, that is: for all $1 \leq j \leq \operatorname{dim} \mathrm{H}$, there exists $\left\{\alpha_{k}^{j} \in \mathrm{C}, k \in\{0,1, \cdots, N-1\}^{p}\right.$ with at least one $\left.\alpha_{k}^{j} \neq 0\right\}$ such that

$$
\Lambda_{N}^{\Gamma}\left(\sum_{k \in\{0,1, \cdots, N-1\}^{p}} \alpha_{k}^{j} \delta_{k} \cdot v_{j}-\delta_{(N-1)+e_{i}} \cdot v_{j}\right)=0
$$

with $\Lambda_{N}^{\Gamma}: F_{N} \times F_{N} \rightarrow \mathrm{C}$ the hspf associated with the kernel $\Gamma_{N}$ as previous. Then, there exists a d-atomic positive operator-valued representing measure $F_{\Gamma}$, with $d=\operatorname{dim}_{\mathrm{C}} K_{N}$ atoms, on a compact set in $\mathrm{R}^{p}$, such that:

$$
\begin{gathered}
\Gamma_{n}=\int_{\mathrm{R}^{p}} t^{n} \mathrm{~d} F_{\Gamma}(t), \forall n=\left(n_{1}, \cdots, n_{p}\right) \in \mathrm{N}^{p}, \\
n_{i} \leq 2 N, 1 \leq i \leq p .
\end{gathered}
$$

Proof. As in Proposition 4.3, in the same conditions 
about the kernel $\Gamma_{N}$, stable at $N-1$ and with the same notations, we obtain a commuting $p$-tuple of selfadjoint operators $A=\left(A_{1}, \cdots, A_{p}\right), \quad A_{i}: K_{N} \rightarrow K_{N}, \quad 1 \leq i \leq p$. From [7], when we aplly Kolmogorov's decomposition theorem to the positive definite kernel $\Gamma_{N}$, we get the representations: $\Gamma_{n+m}=h_{m}^{*} h_{n}$ for every $n, m \in\{0,1, \cdots, N\}^{p^{n+m}}$ with the operators $h_{n}: \mathrm{H} \rightarrow K_{N}$,

$$
\begin{aligned}
h_{n} x & =\widehat{\delta_{n} \cdot x}=\delta_{n} \cdot x+T_{N}, \delta_{n} \cdot x:\{0,1, \cdots, N\}^{p} \\
& \rightarrow \mathrm{H}, \delta_{n k} x=\prod_{i=1}^{p} \delta_{n_{i} k_{i}} x .
\end{aligned}
$$

Accordingly to Remark 4.4, we have:

$A^{m}\left[h_{0} p(m)\right]=h_{m} p(m)$ for every $m \in\{0,1, \cdots, N\}^{p}$ and every arbitrary vector-value $p(m) \in \mathrm{H}$. That is the representations $A^{m} h_{0}=h_{m}$ occur, and by replacing in the above representations, we obtain

$$
\begin{aligned}
& \Gamma_{n+m}=h_{0}^{*} A^{m} \circ A^{n} h_{0}=h_{0}^{*} A^{m+n} h_{0}, \\
& m, n \in \mathrm{N}^{p}, 0 \leq n_{i}, m_{i} \leq N .
\end{aligned}
$$

In these conditions, with respect to the joint spectral measure associated to the commuting tuple $A$, acting on the finite dimensional Hilbert space $K_{N}$, the join spectrum $\Sigma_{A} \subset \sigma\left(A_{1}\right) \times \cdots \times \sigma\left(A_{p}\right)$ with $\sigma\left(A_{k}\right)$ the spectrum of the bounded operator $A_{k}$ defined on $K_{N}$. The set consists only of isolated, in finite number, principal values of $A_{k}$; consequently $\Sigma_{A}$. is an atomic set and the joint spectral measure $E_{A}$ of $A$ is an atomic one. With respect to the joint spectral measure, we have

$$
\begin{aligned}
& \Gamma_{n+m}=h_{0}^{*} A^{m+n} h_{0}=\int_{\mathrm{R}^{p}} t^{n+m} \mathrm{~d} h_{0}^{*} E_{A}(t) h_{0}, \\
& m, n \in \mathrm{N}^{p}, 0 \leq n_{i}, m_{i} \leq N .
\end{aligned}
$$

We denote with $F_{A}(\sigma)=h_{0}^{*} E_{A}(\sigma) h_{0}$, for any $\sigma \in \operatorname{Bor}\left(\Sigma_{A}\right)$ a positive, operator-valued atomic measure and obtained the representations:

$$
\Gamma_{n+m}=\int_{\mathrm{R}^{p}} t^{n+m} \mathrm{~d} F_{A}(t), m, n \in \mathrm{N}^{p}, 0 \leq n_{i}, m_{i} \leq N .
$$

We consider the vector space

$$
\tilde{A}=\left\{p(A)=\sum_{n \in\{0,1, \cdots, l\}^{p}} A^{n}\left[h_{0} p(n)\right], p=\sum_{n \in\{0,1, \cdots, l\}^{p}} \delta_{n} \cdot p(n) \in F_{l}, p, l \geq N, \text { arbitrary }\right\} \subset K_{N}
$$

and define the map $\pi: K_{N} \rightarrow \tilde{A}, \pi(\hat{p})=p(A)$ when

$$
p=\sum_{n \in\{0,1, \cdots, N\}^{p}} \delta_{n} \cdot p(n)
$$

and

$$
\begin{aligned}
0 & =\Lambda_{N}^{\Gamma}\left(p-p_{1}, p-p_{1}\right)=\sum_{l, m \in\{0,1, \cdots, N\}^{p}}\left\langle\Gamma(l, m)\left(p-p_{1}\right)(m),\left(p-p_{1}\right)(l)\right\rangle_{\mathrm{H}} \\
& =\sum_{l, m \in\{0,1, \cdots, N\}^{p}}\left\langle\Gamma_{l+m}\left(p-p_{1}\right)(m),\left(p-p_{1}\right)(l)\right\rangle_{\mathrm{H}} \\
& =\sum_{l, m \in\{0,1, \cdots, N\}^{p}}\left\langle\left[h_{l}\right]^{*}\left[h_{m}\left(p-p_{1}\right)(m)\right],\left(p-p_{1}\right)(l)\right\rangle_{K_{N}} \\
& =\sum_{l, m \in\{0,1, \cdots, N\}^{p}}\left\langle\left[h_{m}\left(p-p_{1}\right)(m)\right],\left[h_{l}\left(p-p_{1}\right)(l)\right]\right\rangle_{K_{N}} \\
& =\sum_{m, l \in\{0,1, \cdots, N\}^{p}}\left\langle A^{m}\left[h_{0}\left(p-p_{1}\right)(m)\right], A^{l}\left[h_{0}\left(p-p_{1}\right)(l)\right]\right\rangle_{K_{N}} \\
& =\left\|\sum_{n \in\{0,1, \cdots, N\}^{p}} A^{n}\left[h_{0}\left(p-p_{1}\right)(n)\right]\right\|_{K_{N}}=0 .
\end{aligned}
$$

That is $p(A)=p_{1}(A)$. From the definition, $\pi$ is linear; we prove that $\pi$ is also injective. Let us consider

$$
\begin{aligned}
& p_{1}=\sum_{n \in\{0,1, \cdots, N\}^{p}} \delta_{n} \cdot p_{1}(n), \\
& p_{2}=\sum_{n \in\{0,1, \cdots, N\}^{p}} \delta_{n} \cdot p_{2}(n) \in F_{N}
\end{aligned}
$$

such that $p_{1}(A)=p_{2}(A) \in \tilde{A}$, that is:

$$
p(A)=\sum_{n \in\{0,1, \cdots, N\}^{p}} A^{n}\left[h_{0} p(n)\right]
$$

check the definition is correct, we shall use again Remark 4.4 and show that, if $p_{1} \in F_{N}$, with $p-p_{1} \in T_{N}$, we have $p_{1}(A)=p(A)$. Indeed, $p-p_{1} \in T_{N}$ means that 


$$
\begin{aligned}
\sum_{n \in\{0,1, \cdots, N\}^{p}} A^{n}\left[h_{n} p_{1}(n)\right] & =\sum_{n \in\{0,1, \cdots, N\}^{p}} A^{n}\left[h_{n} p_{2}(n)\right] \\
& \Leftrightarrow\left\langle\sum_{n \in\{0,1, \cdots, N\}^{p}} A^{n}\left[h_{n}\left(p_{1}-p_{2}\right)(n)\right], \sum_{m \in\{0,1, \cdots, N\}^{p}} A^{m}\left[h_{0}\left(p_{1}-p_{2}\right)(m)\right]\right\rangle_{K_{N}}=0 \\
& \Leftrightarrow\left\langle\sum_{n \in\{0,1, \cdots, \cdots,\}^{p}}\left[h_{n}\left(p_{1}-p_{2}\right)(n)\right], \sum_{m \in\{0,1, \cdots, N\}^{p}}\left[h_{m}\left(p_{1}-p_{2}\right)(m)\right]\right\rangle_{K_{N}}=0 \\
& \Leftrightarrow\left\langle\sum_{n, m \in\{0,1, \cdots, N\}^{p}} h_{m}^{*}\left[h_{n}\left(p_{1}-p_{2}\right)(n)\right],\left(p_{1}-p_{2}\right)(m)\right\rangle_{\mathrm{H}}=0 \\
& \Leftrightarrow \sum_{n, m \in\{0,1, \cdots, N\}^{p}}\left\langle\Gamma_{m+n}\left(p_{1}-p_{2}\right)(n),\left(p_{1}-p_{2}\right)(m)\right\rangle_{\mathrm{H}}=0 \\
& \Leftrightarrow \Lambda_{N}^{\Gamma}\left(p_{1}-p_{2}, p_{1}-p_{2}\right)=0 \Leftrightarrow \hat{p}_{1}=\hat{p}_{2} ;
\end{aligned}
$$

that is $\pi$ is an injective map. We show that $\pi: K_{N} \rightarrow \tilde{A}$ is also a surjective map. We consider the element $q(A)=\sum_{m \in\left\{0,1, \cdots, l^{p}\right.} A^{m}\left[h_{0} q(m)\right], l \geq N$ and prove that there exists $p \in F_{N}$ such that $(p-q) \in T_{l}$. Indeed, $\Lambda_{\infty}$ is stable at any $l \geq N-1$, by recurrence, in Proposition 4.7, we have proved that there exists $p \in F_{N}$ such that $\Lambda_{\infty}(p-q, p-q)=0$ that is

$$
\begin{aligned}
& \left\|p(A)-\sum_{m \in\{0,1, \cdots, l\}^{p}} A^{m}\left[h_{0} q(m)\right]\right\|_{K_{N}} \\
& =0 \Leftrightarrow p(A)=q(A) .
\end{aligned}
$$

The map $\pi$, above defined, is an isomorphism of vectorial spaces, consequently: $\operatorname{dim}_{\mathrm{C}} K_{N}=\operatorname{dim}_{\mathrm{C}} \tilde{A}=d$.

Let also

$$
\tilde{A}_{i}=\left\{\sum_{k \in\{0,1, \cdots, l\}^{p}} \alpha_{k} A^{k}\left[h_{0} v_{i}\right], \alpha_{k} \in \mathrm{C}, l \geq N \text { arbitrary }\right\} \subset K_{N},
$$

with $\left[h_{0} v_{i}\right]=\delta_{0} \cdot v_{i}+T_{N}$. Obviously, $\tilde{A}_{i}$ is a subspace in $\tilde{A}$ (the subspace generated in $K_{N}$ by $A^{k}\left[h_{0} v_{i}\right]$ ). Because of property 2) Proposition 4.10. of the kernel $\Gamma_{N}$, there exists scalars $\left\{\alpha_{j}\right\} \subset \mathrm{C}$ such that

$$
\sum_{j \in\{0,1, \cdots, N-1\}^{p}} \alpha_{j} \delta j \cdot v_{i}+\alpha_{N}^{k} \delta_{(N-1)+e_{k}} \cdot v_{i} \in T_{N} ;
$$

that is

$$
\sum_{j \in\{0,1, \cdots, N-1\}^{p}} \alpha_{j} h_{j} v_{i}+\alpha_{N}^{k} h_{(N-1)+e_{k}} v_{i}=0_{K_{N}}, \alpha_{N}^{k} \neq 0 .
$$

It follows that

$$
\begin{aligned}
& J_{N-1 ; N}^{-1}\left(h_{(N-1)+e_{k}} v_{i}\right)=-\sum_{j \in\{0,1, \cdots, N-1\}^{p}}\left(\alpha_{N}^{k}\right)^{-1} \alpha_{j} h_{j} v_{i} \\
& =-\sum_{j \in\{0,1, \cdots, N-1\}^{p}}\left(\alpha_{N}^{k}\right)^{-1} \alpha_{j} A^{j}\left[h_{0} v_{i}\right] .
\end{aligned}
$$

Because $J_{N-1 ; N}$ is an isomorphism of vectorial spaces, the obtained representation is uniquely determinated (modulo $T_{N}$ ) and it results easily from Remark 4.4). From property 1) of the kernel $\Gamma_{N}$, we have $\tilde{A}_{i} \cap \tilde{A}_{j}=0_{T_{N}}$ whenever $i \neq j$, and also from 2), $\operatorname{dim}_{\mathrm{C}} \tilde{A}_{i}=\operatorname{dim}_{\mathrm{C}} \tilde{A}_{j}=s$ for all $1 \leq i \leq \operatorname{dim}_{\mathrm{C}} \mathrm{H}$. We have proved in this way that $\tilde{A}=\oplus_{i=1}^{\operatorname{dimH}} A_{i} \quad(\oplus$ represents the direct sum. $)$ With the usual operator's multiplication

$A^{m}\left[h_{0} v_{i}\right] \circ A^{n}\left[h_{0} v_{i}\right]=A^{m+n}\left[h_{0} v_{i}\right]$ for all $m, n \in \mathrm{N}^{p}$, and from Remark 4.9, endowed with an echivalent norm induced on $\tilde{A}_{i}$ by the norm on $K_{N}$ via the map $\pi$, the subspaces $\tilde{A}_{i}$ have all a structure of unital commuting $\mathrm{C}^{*}$ algebra with dimension $s=d / \operatorname{dim} \mathrm{H}$, and $d=\operatorname{dim} K_{N}$. In these conditions, the joint spectral measure of

$A_{\left[h_{0} v_{i}\right]}=\left(A_{1}, \cdots, A_{p}\right)_{\left[h_{0} v_{i}\right]}$ has precisely $s$ characters, therefore, the joint spectrum $\Sigma_{A \mid h_{0} v_{i}}$ has exactely $s$ atoms. Because of the representation $\tilde{A}=\oplus_{i=1}^{\operatorname{dim} H} A_{i}$, it follows that the joint spectrum of $A$ has exactely $s \cdot \operatorname{dim} \mathrm{H}=d=$ $\operatorname{dim} K_{N}$ atoms. Consequently the measure $\mathrm{d} F_{A}$, in $\Gamma_{N}$ 's representations, has the same number $d=\operatorname{dim} K_{N}$ of atoms.

\section{Conclusion}

We adapt the concept of "stability of the dimension", in [1], of some Hilbert spaces obtained as the qotient spaces of scalar polynomials of finite degree with respect to the null space of the Riesz functional, to that of "stability of the dimension" of some Hilbert spaces obtained as the quotient spaces of some vectorial-valued functions with respect to the null space of some hermitian square positive functional associated with a positive defined kernel of operators. The stability of this dimension is considered in connection with a truncated operator valued moment problem. The stability of the dimension of the obtained Hilbert space, represents the conditrion for stability of 
the number of atoms of the obtained operator-valued atomic representing measure for the given kernel.

\section{REFERENCES}

[1] F. H. Vasilescu, "Dimension Stability in Truncated Moment Problems," Journal of Mathematical Analysis and Applications, Vol. 388, No. 1, 2012, pp. 219-230. doi:10.1016/j.jmaa.2011.11.063

[2] R. E. Curto and L. A. Fialkow, "Truncated K-Moment Problems in Several Variables," Journal Operator Theory, Vol. 54, No. 1, 2005, pp. 189-226.

[3] R. E. Curto and L. A. Fialkow, "Flat Extension of Positive Moment Matrices. Relation in Analytic or Conjugate Terms," Operator Theory Advanced and Applications, Vol. 104, Birkhäuser Verlag, Basel, 1998, pp. 59-82.
[4] T. Ando, "Truncated Moment Problems for Operators," Acta Scientia Mathematica, Szeged, Vol. 31, 1970, pp. 319-334.

[5] M. Putinar, "Inverse Problems of Perturbation Theory and Moment Problems," Functional Analysis and Related Topics, World Scientific, Singapore City, 1991, pp. 99116.

[6] M. Putinar and F. H. Vasilescu, "Solving Moment Problems by Dimensional Extension," Annals of Mathematics, Vol. 148, No. 3, 1999, pp. 1087-1107. doi: $10.2307 / 121083$

[7] L. Lemnete-Ninulescu, "Truncated Trigonometric and Hausdorff Moment Problems for Operators," An Operator Theory Summer, Proceedings of the 23th International Operator Conference, Timisoara, 29 June-4 July 2010, Theta, 2012, pp. 51-61. 